\title{
Inference of Event-Recording Automata using Timed Decision Trees
}

\author{
Olga Grinchtein \\ IT Department \\ Uppsala University \\ Uppsala, Sweden \\ olgag@it.uu.se \\ Bengt Jonsson \\ IT Department \\ Uppsala University \\ Uppsala, Sweden \\ bengt@it.uu.se
}

\begin{abstract}
In regular inference, the problem is to infer a regular language, typically represented by a deterministic finite automaton (DFA) from answers to a finite set of membership queries, each of which asks whether the language contains a certain word. There are many algorithms for learning DFAs, the most well-known being the $L^{*}$ algorithm due to Dana Angluin. However, there are almost no extensions of these algorithms to the setting of timed systems. We present an algorithm for inferring a model of a timed system using Angluin's setup. One of the most popular model for timed system is timed automata. Since timed automata can freely use an arbitrary number of clocks, we restrict our attention to systems that can be described by event-recording automata (DERAs). In previous work, we have presented an algorithm for inferring a DERA in the form of a region graph. In this paper, we present a novel inference algorithm for DERAs, which avoids constructing a (usually prohibitively large) region graph. We must then develop techniques for inferring guards on transitions of a DERA. Our construction deviates from previous work on inference of DERAs in that it first constructs a so called timed decision tree from observations of system behavior, which is thereafter folded into an automaton.
\end{abstract}

\section{Introduction}

Research during the last decades has developed powerful techniques for using models of reactive systems in specification, automated verification (e.g., [CGP99]), test case generation (e.g., [FJJV97, SEG00]), implementation (e.g., $\left[\mathrm{HLN}^{+}\right.$90]), and validation of reactive systems in telecommunication, embedded control, and related application areas. Typically, such models are assumed to be developed a priori during the specification and design phases of system development. In practice, however, often no formal specification is available, or becomes outdated as the system evolves over time. One must then construct a model that describes the behavior of an existing system or implementation. In software verification, techniques are being developed for generating abstract models of software 
modules by static analysis of source code (e.g., $\left.\left[\mathrm{CDH}^{+} 00, \mathrm{Hol} 00\right]\right)$. However, peripheral hardware components, library modules, or third-party software systems do not allow static analysis. In practice, such systems must be analyzed by observing their external behavior. Techniques for constructing finite-state models by analysis of externally observable behavior (black-box techniques) have been used, e.g., in regression testing [HHNS02, HNS03]), and in model checking [GPY02] of finite-state systems for which no model or source code is available.

The construction of models from observations of system behavior can be seen as a learning problem. For finite-state reactive systems, this can be formulated as the problem of regular inference: to infer a (deterministic) finite automaton by posing a finite set of membership queries, each of which asks whether a certain word is accepted by the automaton or not. There are several slightly different algorithms for regular inference (e.g., [Ang87, Gol67, KV94, RS93, BDG97]), which guarantee that a correct automaton will be constructed if "sufficiently many" membership queries have been posed. In some settings, such as in the $L^{*}$ algorithm [Ang87], the inference algorithm may also pose equivalence queries that ask whether a hypothesized automaton is equivalent to the one that is being investigated: such a query is answered either by yes or by a counterexample on which the hypothesis and the correct automata disagree.

In this paper, we extend the inference algorithm of Angluin and others to the setting of timed systems, more precisely systems that can be described by a timed automaton [AD94], i.e., a finite automaton equipped with clocks that constrain the times of occurrences of actions. One motivation is to develop techniques for creating abstract timed models of hardware components, device drivers, etc., which can then be used in analysis of timed reactive systems. Timed automata have several characteristics that are problematic for the design of inference algorithms: it is not observable how many clocks they use, nor when the clocks are reset, and timed automata can not in general be determinized [AD94]. We therefore restrict consideration to the class of eventrecording automata [AFH99]. These are timed automata that, for every action $a$, use a clock that records the time of the last occurrence of $a$. Event-recording automata can be determinized, and are sufficiently expressive to model many interesting timed systems; for instance, they are as powerful as timed transition systems [HMP94, AFH99], another popular model for timed systems. We assume that an inference algorithm observes a system by checking whether certain actions can be performed at certain moments in time, and that it is able to control and record precisely the timing of the occurrence of each action.

In our earlier work [GJL05], we have designed an inference technique for event-recording automata, which infers explicit representation of the region graph [AD94]. The region graph can be viewed as an ordinary automaton, and be inferred using techniques for untimed regular inference, but at the cost of a high blow-up: for instance, each transition will be enabled in only one clock region causing an explosion in the number of generated transitions. In this paper, we avoid the region graph construction; one goal is that guards on transitions should not depend on clocks that are not relevant for the occurrence 
of current or future actions. We must then develop techniques for inferring "relevant" guards on transitions of a timed automaton.

A novel feature of our algorithm (in comparison with other regular inference algorithms) is that it contains two constructions: the first construction generates a so-called timed decision tree from the answers to the membership queries that have been performed so far. The conditions (or guards) that distinguish between branches in this tree should correspond to guards in the inferred automaton. A guard is therefore introduced only if there is a pair of observations with different outcomes, where this guard is the only means to distinguish between them. The algorithm includes a systematic search for such pairs of observations. The second construction consists in folding the timed decision tree into an eventrecording automaton by appropriate merging of nodes, in an analogous manner as in algorithms for (untimed) regular inference.

Related Work In previous work, authors of this paper [GJL04] have presented algorithms for the restricted class of event-recording automata that have at most one outgoing transition per action; under this restriction, the time of an action occurrence depends only on the (untimed) past sequence of actions, and not on their timing. Later [GJL05] the same authors presented an algorithm for inferring of the full class of event-recording automata. This is much more challenging, since it must be inferred how the timing of an action occurrence may depend on timing of previous actions. A drawback of the algorithm is that it constructs a region graph, thus causing an explosion in the number of states and transitions.

Verwer et. al [VdWW06] present an algorithm for passive learning of timed automata with one clock which is reset at every transition. In passive learning an automaton is constructed from a given set of observations and no queries are performed. Passive learning even for this class of timed automata is a hard problem, since one must decide how to introduce guards in the automaton. The algorithm constructs a prefix tree from a timed sample and then tries to merge nodes of this tree pairwise to form an automaton. If the resulting automaton does not agree with the sample then the last merge is undone and a new merge is attempted. The algorithm does not construct timed automata in a systematic way, and it is hard to generalize the algorithm to timed automata with more than one clock.

A related field to timed automata learning is conformance testing for realtime systems. It was shown in $\left[\right.$ BGJ $\left.^{+} 05\right]$ that if a set of examples form a conformance test suite for a finite state machine $M$, then $M$ can be inferred from these set of examples using automata learning techniques. Conversely, it was also shown that if a finite state machine $M$ is inferred from a set of examples, and furthermore $M$ is the only such automaton, then the set of examples forms a conformance test suite for $M$. Springintveld et al. [SVD01] introduce an algorithm which generates a conformance test suite for timed automata. The algorithm constructs a so-called grid automaton, which is obtained by discretizing the time domain with the help of time transitions, each of which advances 
time by $2^{-n}$ time units for some sufficiently large natural number $n$. Then the Vasilevskii-Chow algorithm [Cho78], [Vas73] is applied to the grid automaton to generate a conformance test suite.

Several papers are concerned with finding a definition of timed languages which is suitable as a basis for inference algorithms. There are several works that define determinizable classes of timed automata (e.g., [AFH99, SV96]) and right-congruences of timed languages (e.g., [MP04, HRS98, Wil94]), motivated by testing and verification.

Structure of Paper The paper is structured as follows. After preliminaries and a definition of event-recording automata in the next section, the construction and maintenance of timed decision trees is described in Sections 4 and 5, followed by a technique for folding them into automata in Section 6. The overall algorithm is summarized in Section 7, accompanied by an analysis of the query complexity of our algorithm. An illustrating example is given in Section 8, and finally Section 9 concludes the paper.

\section{Preliminaries}

We write $\mathbb{R}^{\geq 0}$ for the set of nonnegative real numbers, and $\mathbb{N}$ for the set of natural numbers. Let $\Sigma$ be a finite alphabet of size $|\Sigma|$. A timed word over $\Sigma$ is a finite sequence $w_{t}=\left(a_{1}, t_{1}\right)\left(a_{2}, t_{2}\right) \ldots\left(a_{n}, t_{n}\right)$ of symbols $a_{i} \in \Sigma$ paired with nonnegative real numbers $t_{i} \in \mathbb{R}^{\geq 0}$ such that the sequence $t_{1} t_{2} \ldots t_{n}$ of time-stamps is nondecreasing. We use $\lambda$ to denote the empty word and $\left|w_{t}\right|$ to denote the number of pairs in $w_{t}$.

An event-recording automaton contains for every symbol $a \in \Sigma$ a clock $x_{a}$, called the event-recording clock of $a$. Intuitively, $x_{a}$ records the time elapsed since the last occurrence of the symbol $a$. We write $C_{\Sigma}$ for the set $\left\{x_{a} \mid a \in \Sigma\right\}$ of event-recording clocks. A clock valuation $\gamma$ is a mapping from $C_{\Sigma}$ to $\mathbb{R}^{\geq 0}$. We define $\gamma\left[x_{a} \rightarrow 0\right]$ to be the clock valuation $\gamma^{\prime}$ such that $\gamma^{\prime}\left(x_{a}\right)=0$ and $\gamma^{\prime}\left(x_{b}\right)=\gamma\left(x_{b}\right)$ for all $b \neq a$.

Throughout the paper, we will use an alternative, equivalent representation of timed words, namely clocked words. A clocked suffix word over $\Sigma$ is a sequence $w_{c}=\left(a_{1}, \gamma_{1}\right)\left(a_{2}, \gamma_{2}\right) \ldots\left(a_{n}, \gamma_{n}\right)$ of symbols $a_{i} \in \Sigma$ that are paired with clock valuations, which for all $a \in \Sigma$ satisfies

$$
\gamma_{i}\left(x_{a}\right)=\gamma_{i-1}\left(x_{a}\right)+\gamma_{i}\left(x_{a_{i-1}}\right) \text { whenever } 1<i \leq n \text { and } a \neq a_{i-1} .
$$

A clocked word over $\Sigma$ is a clocked suffix word $w_{c}=\left(a_{1}, \gamma_{1}\right)\left(a_{2}, \gamma_{2}\right) \ldots\left(a_{n}, \gamma_{n}\right)$ which in addition satisfies

$$
\gamma_{1}\left(x_{a}\right)=\gamma_{1}\left(x_{b}\right) \text { for all } a, b \in \Sigma .
$$

Each timed word $\left(a_{1}, t_{1}\right)\left(a_{2}, t_{2}\right) \ldots\left(a_{n}, t_{n}\right)$ can be naturally transformed into the clocked word $\left(a_{1}, \gamma_{1}\right)\left(a_{2}, \gamma_{2}\right) \ldots\left(a_{n}, \gamma_{n}\right)$ where for each $i$ with $1 \leq i \leq n$,

- $\gamma_{i}\left(x_{a}\right)=t_{i}$ if $a_{j} \neq a$ for $1 \leq j<i$, 
- $\gamma_{i}\left(x_{a}\right)=t_{i}-t_{j}$ if there is a $j$ with $1 \leq j<i$ and $a_{j}=a$, such that $a_{k} \neq a$ for $j<k<i$ (i.e. time elapsed since last occurrence of the symbol $a$ in the timed word).

Conversely, each clocked word can also be transformed into the timed word. A timed language over $\Sigma$ is a set of timed words (or equivalently clocked words) over $\Sigma$.

A clock constraint is a conjunction of atomic constraints of the form $x \sim k$ or $x-y \sim k$ for $x, y \in C_{\Sigma}, \sim \in\{<, \leq,>, \geq\}$, and $k \in \mathbb{N}$. A clock constraint is $K$-bounded if it contains no constant larger than $K$. We use $\gamma \models \varphi$ to denote that the clock valuation $\gamma$ satisfies the clock constraint $\varphi$, defined in the usual manner. A clock guard is a clock constraint whose conjuncts are only of the form $x \sim k$ (for $\left.x \in C_{\Sigma}, \sim \in\{<, \leq\rangle,, \geq\right\}$ ), i.e., comparison between clocks is not permitted. The set of clock guards is denoted by $G_{\Sigma}$. A $K$-bounded simple clock guard is a clock constraint whose conjuncts are only of the form $x=k$, $k^{\prime}<x<k^{\prime}+1$ or $x>K$ for $x \in C_{\Sigma}, 0 \leq k \leq K$ and $0 \leq k^{\prime} \leq K-1$.

A guarded word is a sequence $w_{g}=\left(a_{1}, g_{1}\right)\left(a_{2}, g_{2}\right) \ldots\left(a_{n}, g_{n}\right)$ of symbols $a_{i} \in \Sigma$ that are paired with clock guards. For a clocked suffix word $w_{c}=$ $\left(a_{1}, \gamma_{1}\right)\left(a_{2}, \gamma_{2}\right) \ldots\left(a_{n}, \gamma_{n}\right)$ we use $w_{c} \models w_{g}$ to denote that $\gamma_{i} \models g_{i}$ for $1 \leq i \leq n$. We say that $w_{c}$ leads to $w_{g}$ or that $w_{c}$ is in $w_{g}$ if $w_{c} \vDash w_{g}$. We say that $w_{c}$ passes $w_{g}$ if $w_{c}^{\prime} \vDash w_{g}$ for some prefix $w_{c}^{\prime}$ of $w_{c}$. If for all $i, g_{i}$ is a $K$-bounded simple clock guard, $w_{g}$ is called $K$-bounded simple guarded word. We omit $K$-bounded if $K$ is clear from the context.

For a guarded word $w_{g}$, we introduce the strongest postcondition of $w_{g}$, denoted by $s p\left(w_{g}\right)$, as the constraint on clock values that are induced by $w_{g}$ on any following occurrence of a symbol. Postcondition computation is central in techniques for symbolic verification of timed automata $\left[\mathrm{BDM}^{+} 98, \mathrm{BLL}^{+} 96\right]$, and can be done inductively as follows:

- $\operatorname{sp}(\lambda)=\bigwedge_{a, b \in \Sigma} x_{a}=x_{b}$,

- $s p\left(w_{g}(a, g)\right)=\left(\left(s p\left(w_{g}\right) \wedge g\right)\left[x_{a} \mapsto 0\right]\right) \uparrow$,

where for clock constraint $\varphi$ and clock $x$,

- $\varphi[x \mapsto 0]$ is the condition $x=0 \wedge \exists x . \varphi$,

- $\varphi \uparrow$ is the condition $\exists d \cdot \varphi^{\prime}$, where $d$ ranges over $\mathbb{R}^{\geq 0}$ and where $\varphi^{\prime}$ is obtained from $\varphi$ by replacing each clock $y$ by $y-d$.

Both operations can be expressed as corresponding operations on clock constraints [DT98].

For each clock constraint $\varphi$ there are in general several other clock constraints that are equivalent to $\varphi$ in the sense that they identify the same set of clock valuations. If $\varphi$ is satisfiable, there is among these a unique canonical clock constraint, denoted $\operatorname{Can}(\varphi)$, obtained by closing $\varphi$ under all consequences of pairs of conjuncts in $\varphi$, i.e.,

- from two difference bounds, such as $x_{a}-x_{b} \leq 2$ and $x_{b}-x_{c}<3$, we derive a new difference bound, viz. $x_{a}-x_{c}<5$, and 
- from a difference bound and a clock bound, such as $x_{a}-x_{b} \leq 2$ and $x_{a} \geq 3$, we derive a new clock bound, viz. $x_{b} \geq 1$,

- from an upper and a lower clock bound, such as $x_{a} \leq 3$ and $x_{b}>2$, we derive a new difference bound, viz. $x_{a}-x_{b}<1$,

until saturation, and thereafter keeping the tightest bounds for each clock and each clock difference. If the canonical form contains inconsistent constraints, or requires some clock to be negative, then the clock constraint is unsatisfiable. We define the canonical form for an unsatisfiable clock constraint to be false [Dil89].

For $t \in \mathbb{R}^{\geq 0}$, fract $(t)$ denotes the fractional part of $t,\lfloor t\rfloor$ denotes the integer part of $t$, and $\lceil t\rceil=\lfloor t\rfloor+1$. Given a positive non-negative $K$, we define the region equivalence, denoted $\sim_{K}$, on the set of clock valuations by $\gamma \sim_{K} \gamma^{\prime}$ if

- for all $x_{a} \in C_{\Sigma}$, either

$$
\begin{aligned}
& -\gamma\left(x_{a}\right) \text { and } \gamma^{\prime}\left(x_{a}\right) \text { are both greater than } K \text {, or } \\
& -\left\lfloor\gamma\left(x_{a}\right)\right\rfloor=\left\lfloor\gamma^{\prime}\left(x_{a}\right)\right\rfloor \text { and } \operatorname{fract}\left(\gamma\left(x_{a}\right)\right)=0 \text { iff } \operatorname{fract}\left(\gamma^{\prime}\left(x_{a}\right)\right)=0,
\end{aligned}
$$

and

- for all $x_{a}, x_{b} \in C_{\Sigma}$ with $\gamma\left(x_{a}\right) \leq K$ and $\gamma\left(x_{b}\right) \leq K$, $\operatorname{fract}\left(\gamma\left(x_{a}\right)\right) \leq \operatorname{fract}\left(\gamma\left(x_{b}\right)\right)$ iff $\operatorname{fract}\left(\gamma^{\prime}\left(x_{a}\right)\right) \leq \operatorname{fract}\left(\gamma^{\prime}\left(x_{b}\right)\right)$.

A region is an equivalence class of clock valuations induced by $\sim_{K}$. We denote by $[\gamma]_{K}$ the region of $\gamma$. The number of regions $R_{K}$ is bounded by $|\Sigma| ! 2^{|\Sigma|}(2 K+$ $2)^{|\Sigma|}$ [AD94]. A region $[\gamma]_{K}$ is initial if $\gamma\left(x_{a}\right)=0$ for some $a \in \Sigma$.

Region equivalence induces a natural equivalence on clock constraints. We say that two clock constraints $\varphi$ and $\varphi^{\prime}$ are region-equivalent, denoted $\varphi \approx_{K} \varphi^{\prime}$, if for each clock valuation $\gamma$ with $\gamma \vDash \varphi$ there is a clock valuation $\gamma^{\prime}$ with $\gamma^{\prime} \vDash \varphi^{\prime}$ such that $\gamma \sim_{K} \gamma^{\prime}$, and vice versa.

Definition 2.1 An event-recording automaton (ERA) over $\Sigma$ is a tuple $\mathcal{A}=$ $\left\langle L, L^{0}, L^{f}, E\right\rangle$ consisting of a finite set $L$ of locations, a set $L^{0} \subseteq L$ of start locations, a set $L^{f}$ of accepting locations, and a finite set $E$ of edges. Each edge is a quadruple $\left(l, l^{\prime}, a, g\right)$ with a source location $l \in L$, a target location $l^{\prime} \in L$, an input symbol $a \in \Sigma$, and a clock guard $g \in G_{\Sigma}$.

We write $l \stackrel{(a, g)}{\rightarrow} l^{\prime}$ to denote $\left(l, l^{\prime}, a, g\right) \in E$.

For a guarded word $w_{g}=\left(a_{1}, g_{1}\right) \ldots\left(a_{n}, g_{n}\right)$, we use $l_{0} \stackrel{w_{g}}{\Rightarrow} l_{n}$ to denote that there is a sequence

$$
l_{0} \stackrel{\left(a_{1}, g_{1}\right)}{\rightarrow} l_{1} \stackrel{\left(a_{2}, g_{2}\right)}{\rightarrow} l_{2} \ldots \stackrel{\left(a_{n-1}, g_{n-1}\right)}{\rightarrow} l_{n-1} \stackrel{\left(a_{n}, g_{n}\right)}{\rightarrow} l_{n}
$$

of locations $l_{i} \in L$ and edges $\left(l_{i-1}, l_{i}, a_{i}, g_{i}\right) \in E$. For a location $l \in L$, let $\mathcal{L}_{g}^{+}(\mathcal{A}, l)$ be the set of guarded words $w_{g}$ such that $l \stackrel{w_{g}}{\Rightarrow} l^{\prime}$ for some accepting $l^{\prime} \in L^{f}$, and let $\mathcal{L}_{g}^{-}(\mathcal{A}, l)$ be the set of guarded words $w_{g}$ such that $l \stackrel{w_{g}}{=} l^{\prime}$ for 
some nonaccepting $l^{\prime} \notin L^{f}$. Let $\mathcal{L}_{c}^{+}(\mathcal{A}, l)$ be the set of clocked suffix words $w_{c}$ such that $w_{c} \vDash w_{g}$ for some $w_{g} \in \mathcal{L}_{g}^{+}(\mathcal{A}, l)$. The clocked word $w_{c}$ is accepted by $\mathcal{A}$ if $w_{c} \in \mathcal{L}_{c}^{+}\left(\mathcal{A}, l^{0}\right)$ where $l^{0} \in L^{0}$, otherwise $w_{c}$ is rejected by $\mathcal{A}$. The timed language $\mathcal{L}(\mathcal{A})$ defined by $\mathcal{A}$ consists of all clocked words accepted by $\mathcal{A}$. Two ERAs $\mathcal{A}$ and $\mathcal{A}^{\prime}$ are equivalent if $\mathcal{L}(\mathcal{A})=\mathcal{L}\left(\mathcal{A}^{\prime}\right)$.

An ERA is time-deterministic (TDERA) iff it has a single start location and for each location $l$ and input symbol $a$, the guards $g_{1}, \ldots, g_{n}$ of edges from $l$ labeled $a$ are total and mutually exclusive, i.e., $g_{1} \vee \ldots \vee g_{n} \equiv$ true and $g_{i} \wedge g_{j} \equiv$ false whenever $1 \leq i<j \leq n$.

\section{Overview of Our Inference Algorithm}

The algorithm presented in this paper is designed to infer a TDERA which is equivalent to a given TDERA $\mathcal{A}$. In this algorithm, which follows the setup of Angluin's algorithm [Ang87], a so called Learner, who initially knows $\Sigma$ and the greatest constant $K$ appearing in clock guards of $\mathcal{A}$, is trying to learn $\mathcal{L}(\mathcal{A})$ by asking queries to a Teacher, who knows $\mathcal{A}$. There are two kinds of queries:

- A membership query consists in asking whether a clocked word $w_{c}$ over $\Sigma$ is in $\mathcal{L}(\mathcal{A})$.

- An equivalence query consists in asking whether a hypothesized TDERA $\mathcal{H}$ is correct, i.e., whether $\mathcal{L}(\mathcal{H})=\mathcal{L}(\mathcal{A})$. The Teacher will answer yes if $\mathcal{H}$ is correct, or else supply a counterexample, i.e, a clocked word $w_{c}$ which is either in $\mathcal{L}(\mathcal{A}) \backslash \mathcal{L}(\mathcal{H})$ or in $\mathcal{L}(\mathcal{H}) \backslash \mathcal{L}(\mathcal{A})$.

The typical behavior of a Learner is to start by asking a sequence of membership queries, and gradually build a hypothesized TDERA $\mathcal{H}$ using the obtained answers. When the Learner feels that she has built a hypothesis $\mathcal{H}$, she makes an equivalence query to find out whether $\mathcal{H}$ is correct. If the answer is yes, the Learner has succeeded, otherwise she uses the returned counterexample to revise $\mathcal{H}$ and perform subsequent membership queries until arriving at a new hypothesized TDERA, etc. The algorithm must guarantee that there is a bound, which depends on $\mathcal{A}$, on the number of performed membership and equivalence queries needed before the Learner is able to make a successful equivalence query.

Let us represent the information gained by the Learner at any point during the learning process as a partial mapping $O b s$ from clocked words to $\{+,-\}$, where + stands for accepted and - for rejected. The domain Dom(Obs) of Obs is the set of clocked words for which membership queries have been performed, or which have been supplied as counterexamples in response to equivalence queries. We will consider the case where $\mathrm{Dom}(\mathrm{Obs})$ is initially empty, but our techniques work equally well when $\operatorname{Dom}(\mathrm{Obs})$ is some given initial set of observations. An inference algorithm should prescribe how, starting from any given mapping Obs, additional membership queries are performed in order to gather sufficient information for constructing a "good hypothesis" in the form of a TDERA $\mathcal{H}$, which can be supplied in an equivalence query. A natural requirement on the 
constructed TDERA $\mathcal{H}$ is that it agrees with $O b s$, in the sense that any clocked word $w_{c} \in \operatorname{Dom}(\mathrm{Obs})$ is accepted by $\mathcal{H}$ if $O b s\left(w_{c}\right)=+$, and rejected by $\mathcal{H}$ if $\operatorname{Obs}\left(w_{c}\right)=-$.

During the gathering of information by membership queries, our algorithm organizes the information in Obs in a so-called timed decision tree (defined in the next section), which roughly can be regarded as a prefix of the "unrolling" of the (later) constructed hypothesized TDERA $\mathcal{H}$ into an infinite tree. A main problem when constructing the timed decision tree is to find suitable guards; these should ideally be the guards that occur on the corresponding edges of the TDERA $\mathcal{A}$. However, the Learner does not know a priori which guards to use in the final TDERA. Therefore, guards will be introduced "by need", when the information in $\mathrm{Obs}$ shows that they are necessary. Initially, the edges in the tree will use only true in guards. Any non-trivial guard in the timed decision tree will be obtained from a pair of clocked words in $\operatorname{Dom}(\mathrm{Obs})$, one of which is rejected and one of which is accepted, and which are so "close" that the obtained guard is the only means to separate them.

The goal of the information gathering through membership queries is that $O b s$ and the timed decision tree should attain four desirable properties: wellformedness and consistency, defined in Section 4, and suffix-completeness and region-consistency, defined in Section 6. When these properties have been attained, the tree will be folded into a hypothesized TDERA by merging nodes which have "similar" future behavior according to Obs and the tree, as will be described in Section 6.

\section{Timed Decision Trees.}

In this section, we present timed decision trees, which are used to organize the results of queries.

Definition 4.1 A timed decision tree is a prefix-closed finite set $\mathcal{N}$ of guarded words, such that for any guarded word $w_{g} \in \mathcal{N}$ and symbol a, the set of guarded words $w_{g}\left(a, g_{1}\right), \ldots, w_{g}\left(a, g_{n}\right)$ in $\mathcal{N}$ that extend $w_{g}$ by the symbol a is either empty, or has the properties that $g_{1} \vee \cdots \vee g_{n} \equiv$ true and $g_{i} \wedge g_{j} \equiv$ false whenever $i \neq j$.

A timed decision tree $\mathcal{N}$ is intended to be seen as a prefix of an unfolding of a TDERA. Each guarded word in $\mathcal{N}$ can then be viewed as the sequence of labels on a sequence of edges from the initial location of the TDERA to some location in its unfolding. We will often refer to the elements of a timed decision tree as nodes. The structure into which results of queries are organized is obtained by combining a timed decision tree with the results of performed queries.

Definition 4.2 An observation structure is a pair $\langle O b s, \mathcal{N}\rangle$, where Obs is a partial mapping from clocked words to $\{+,-\}$ and $\mathcal{N}$ is a timed decision tree. 
The mapping in an observation structure records whether individual clocked words are accepted or rejected, but we extend it to a mapping from nodes in a timed decision tree, as follows. For a partial mapping $O b s$ from clocked words to $\{+,-\}$ and a guarded word $w_{g}$, define

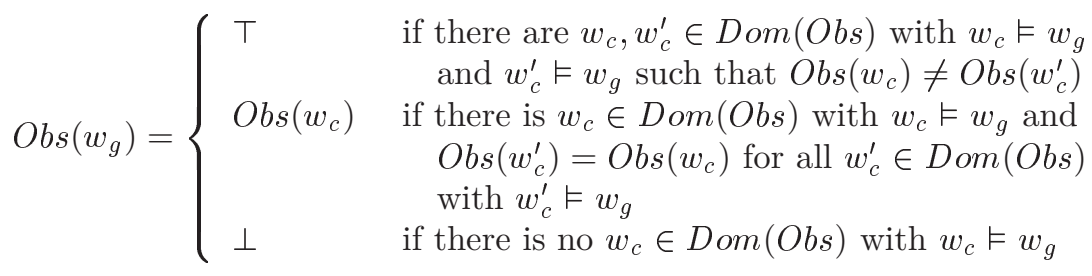

By this definition, the function $O b s$ is overloaded so that it can also be regarded as a labeling of nodes in a timed decision tree. A node is labeled by + (or - ) if every observation that leads to the node is accepted (or rejected). A node is labeled by $\mathrm{T}$, and called inconsistent, (w.r.t. Obs), if there are both accepted and rejected observations that lead to the node. We say that Obs distinguishes between $w_{c}$ and $w_{c}^{\prime}$ if $O b s\left(w_{c}\right) \neq O b s\left(w_{c}^{\prime}\right)$. If there are no observations that lead to the node, the node is labeled by $\perp$.

Definition 4.3 An observation structure $\langle O b s, \mathcal{N}\rangle$ is

- well-formed if

$$
\begin{aligned}
& \text { - for any } w_{c} \in \operatorname{Dom}(\text { Obs }) \text { there is a } w_{g} \in \mathcal{N} \text { such that } w_{c} \vDash w_{g} \text {, and } \\
& -\operatorname{Obs}\left(w_{g}\right) \neq \perp \text { for all } w_{g} \in \mathcal{N},
\end{aligned}
$$

- consistent if $\mathcal{N}$ has no inconsistent nodes w.r.t. Obs.

In the following, we will assume that all observation structures are well-formed, since it is easy to make them well-formed either by adding new nodes that extend parents by suffixes of form $\left(a_{1}\right.$, true $) \cdots\left(a_{m}\right.$, true $)$, or by asking additional membership queries, e.g., for prefixes of clocked words in Dom(Obs). Making an observation structure consistent is more complicated. It typically involves asking additional membership queries to find new guards, and restructuring of the tree, as described in Section 5.

Example 4.4 Figure 1 shows an example of a well-formed observation structure. To the left is the mapping $O b s$; to make the notation more compact we use timed words as a short representation of clocked words. To the right is the timed decision tree. We label the edges of the timed decision tree with pairs of the form $(a, g)$ where $a$ is a symbol and $g$ is a clock guard. The guarded word that constitutes a node is the sequence of labels on the path reaching the node. Each node $w_{g}$ in the tree is labeled by $\operatorname{Obs}\left(w_{g}\right)$.

The only nontrivial guards occur on the two rightmost edges, which separate occurrences of the symbol $b$ depending on whether $x_{a}=0$ or $x_{a}>0$. This guard is motivated by the observations for the two timed words $(a, 0.5)(b, 0.5)$ and 

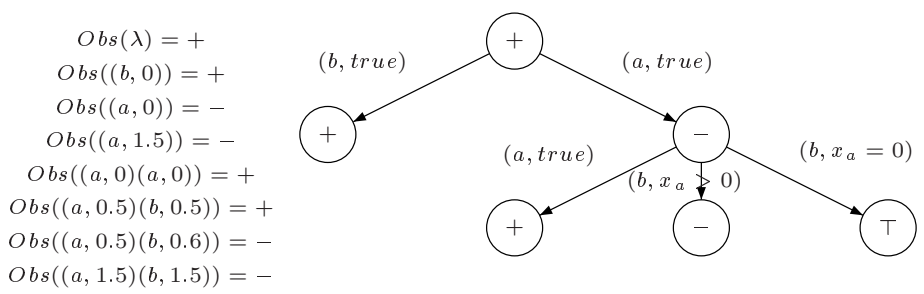

Figure 1: An observation structure $\langle O b s, \mathcal{N}\rangle$, with $O b s$ to the left and $\mathcal{N}$ to the right.

$(a, 0.5)(b, 0.6)$ which are distinguished by Obs. The rightmost node $(a$, true $)\left(b, x_{a}=\right.$ $0)$ is inconsistent since two timed words $(a, 0.5)(b, 0.5)$ and $(a, 1.5)(b, 1.5)$, which are distinguished by $\mathrm{Obs}$, lead to it.

In the next section, we describe how inconsistent nodes, such as the rightmost one in Figure 1, are removed by finding new guards and restructuring the tree after having performed additional membership queries.

\section{$5 \quad$ Removing Inconsistent nodes}

In this section, we describe our procedure for removing inconsistent nodes in a timed decision tree by finding new guards and restructuring the tree accordingly. New guards are inferred from so-called critical pairs, described in Section 5.1, which are pairs of "close" clocked words which are distinguished by Obs and uniquely suggest a guard that separates them, as described in Section 5.2. In Section 5.3 we present a procedure, inspired by binary search, for finding critical pairs whenever the tree contains an inconsistent node, and in Section 5.4 we describe how to restructure the tree to avoid the initial inconsistency.

\subsection{Critical Pairs}

Critical pairs are pairs of "close" clocked words, used to suggest guards in a timed decision tree. To define them, we first formalize the notion of "close" observations. For two nonnegative real numbers $p, p^{\prime} \in \mathbb{R}^{\geq 0}$, define $p \approx_{K} p^{\prime}$ if either

- $p>K$ and $p^{\prime}>K$, or

- both $p$ and $p^{\prime}$ are integers with $p=p^{\prime}$, or

- both $p$ and $p^{\prime}$ are non-integer with $\lfloor p\rfloor=\left\lfloor p^{\prime}\right\rfloor$ (i.e., they have the same integer parts). 
For two clock valuations $\gamma$ and $\gamma^{\prime}$, define $\gamma \approx_{K} \gamma^{\prime}$ if $\gamma\left(x_{a}\right) \approx_{K} \gamma^{\prime}\left(x_{a}\right)$ for all $a \in \Sigma$. For two clocked (suffix) words $w_{c}=\left(a_{1}, \gamma_{1}\right) \ldots\left(a_{n}, \gamma_{n}\right)$ and $w_{c}^{\prime}=$ $\left(a_{1}^{\prime}, \gamma_{1}^{\prime}\right) \ldots\left(a_{n}^{\prime}, \gamma_{n}^{\prime}\right)$, define $w_{c} \approx_{K} w_{c}^{\prime}$ if $a_{i}=a_{i}^{\prime}$ and $\gamma_{i} \approx_{K} \gamma_{i}^{\prime}$ for all $1 \leq i \leq n$.

Definition 5.1 A pair $\left(w_{c}, w_{c}^{\prime}\right)$ of clocked words, where $w_{c}=\left(a_{1}, \gamma_{1}\right) \ldots\left(a_{n}, \gamma_{n}\right)$ and $w_{c}^{\prime}=\left(a_{1}, \gamma_{1}^{\prime}\right) \ldots\left(a_{n}, \gamma_{n}^{\prime}\right)$ is adjacent if $w_{c} \not_{K} w_{c}^{\prime}$, and for all $i$ with $1 \leq i \leq n$ and $a \in \Sigma$, either $\gamma_{i}\left(x_{a}\right)>K$ and $\gamma_{i}^{\prime}\left(x_{a}\right)>K$, or

- whenever $\gamma_{i}^{\prime}\left(x_{a}\right)$ is an integer, then $\gamma_{i}\left(x_{a}\right)=\gamma_{i}^{\prime}\left(x_{a}\right)$,

- whenever $\gamma_{i}\left(x_{a}\right)$ is an integer, and $\gamma_{i}^{\prime}\left(x_{a}\right)$ is not, then $\left|\gamma_{i}\left(x_{a}\right)-\gamma_{i}^{\prime}\left(x_{a}\right)\right|<1$,

- whenever $\gamma_{i}\left(x_{a}\right)$ is not an integer, then $\gamma_{i}\left(x_{a}\right) \approx_{K} \gamma_{i}^{\prime}\left(x_{a}\right)$.

Intuitively, a pair $\left(w_{c}, w_{c}^{\prime}\right)$ of clocked words is adjacent if, for each position $i$ and symbol $a$ for which $\gamma_{i}\left(x_{a}\right) \not_{K} \gamma_{i}^{\prime}\left(x_{a}\right)$, there is exactly one atomic guard which can separate $\gamma_{i}\left(x_{a}\right)$ from $\gamma_{i}^{\prime}\left(x_{a}\right)$. If there is only one position $i$ and symbol $a$ with $\gamma_{i}\left(x_{a}\right) \not \varpi_{K} \gamma_{i}^{\prime}\left(x_{a}\right)$, then this atomic guard is unique, but in general there can be several such positions and symbols. Note that adjacency is not a symmetric relation, i.e., if $\left(w_{c}, w_{c}^{\prime}\right)$ is adjacent, then $\left(w_{c}^{\prime}, w_{c}\right)$ is not adjacent.

As a measure of "closeness" between clocked words in adjacent pairs $\left(w_{c}, w_{c}^{\prime}\right)$, we define $\operatorname{diff}\left(w_{c}, w_{c}^{\prime}\right)$ to be the set of quadruples $\left(i, x_{a}, k, \geq\right)$ and $\left(i, x_{a}, k, \leq\right)$ with $k \leq K$, such that

- $\left(i, x_{a}, k, \geq\right) \in \operatorname{diff}\left(w_{c}, w_{c}^{\prime}\right)$ iff $\gamma_{i}\left(x_{a}\right)=k$ and $\gamma_{i}^{\prime}\left(x_{a}\right)<k$ and

- $\left(i, x_{a}, k, \leq\right) \in \operatorname{diff}\left(w_{c}, w_{c}^{\prime}\right)$ iff $\gamma_{i}\left(x_{a}\right)=k$ and $\gamma_{i}^{\prime}\left(x_{a}\right)>k$.

Example 5.2 As an illustration of Definition 5.1, $\left(w_{c}, w_{c}^{\prime}\right)$ is an adjacent pair when

$$
\begin{aligned}
& w_{c}=\left(a,\left(\begin{array}{l}
x_{a} \rightarrow 0.8 \\
x_{b} \rightarrow 0.8
\end{array}\right)\right)\left(b,\left(\begin{array}{c}
x_{a} \rightarrow 0.2 \\
x_{b} \rightarrow 1.0
\end{array}\right)\right) \text { and } \\
& w_{c}^{\prime}=\left(a,\left(\begin{array}{l}
x_{a} \rightarrow 0.8 \\
x_{b} \rightarrow 0.8
\end{array}\right)\right)\left(b,\left(\begin{array}{c}
x_{a} \rightarrow 0.3 \\
x_{b} \rightarrow 1.1
\end{array}\right)\right) .
\end{aligned}
$$

We have $\operatorname{diff}\left(w_{c}, w_{c}^{\prime}\right)=\left\{\left(2, x_{b}, 1, \leq\right)\right\}$. If $O b s$ distinguishes $w_{c}$ and $w_{c}^{\prime}$, then it is clear that the tree must contain the conjuncts $x_{b}>1$ and $x_{b} \leq 1$ in guards on two different outgoing edges from the node to which $\left(a,\left(\begin{array}{cc}x_{a} & \rightarrow 0.8 \\ x_{b} & \rightarrow 0.8\end{array}\right)\right)$ leads, in order to separate the observations.

Example 5.3 A more complicated example of an adjacent pair is $\left(w_{c}, w_{c}^{\prime}\right)$ where

$$
\begin{aligned}
& w_{c}=\left(a,\left(\begin{array}{l}
x_{a} \rightarrow 1.0 \\
x_{b} \rightarrow 1.0
\end{array}\right)\right)\left(b,\left(\begin{array}{c}
x_{a} \rightarrow 0.5 \\
x_{b} \rightarrow 1.5
\end{array}\right)\right) \text { and } \\
& w_{c}^{\prime}=\left(a,\left(\begin{array}{l}
x_{a} \rightarrow 0.5 \\
x_{b} \rightarrow 0.5
\end{array}\right)\right)\left(b,\left(\begin{array}{c}
x_{a} \rightarrow 0.6 \\
x_{b} \rightarrow 1.1
\end{array}\right)\right)
\end{aligned}
$$

Here $\operatorname{diff}\left(w_{c}, w_{c}^{\prime}\right)=\left\{\left(1, x_{a}, 1, \geq\right),\left(1, x_{b}, 1, \geq\right)\right\}$. If $O b s$ distinguishes $w_{c}$ and $w_{c}^{\prime}$, we can choose either the guard $x_{a} \geq 1$ or the guard $x_{b} \geq 1$ on edges that leave the root, in order to separate the two observations. 
In situations like those of Example 5.3, we should try to obtain more information about $\mathcal{A}$ to resolve the choice between the two guards, by asking more membership queries for clocked words that are very similar to $w_{c}$ and $w_{c}^{\prime}$, hoping to find an adjacent pair $\left(u_{c}, u_{c}^{\prime}\right)$ of clocked words such that $\operatorname{diff}\left(u_{c}, u_{c}^{\prime}\right) \subset \operatorname{diff}\left(w_{c}, w_{c}^{\prime}\right)$, where $\subset$ denotes strict set inclusion. We formalize which clocked words are "very similar" to $w_{c}$ by defining neighbourhood $\left(w_{c}\right)$ as the set of clocked words $w_{c}^{\prime}$ such that $\left(w_{c}, w_{c}^{\prime}\right)$ is an adjacent pair. Letting $w_{c}=\left(a_{1}, \gamma_{1}\right) \ldots\left(a_{n}, \gamma_{n}\right)$, this means that neighbourhood $\left(w_{c}\right)$ is the set of clocked words $\left(a_{1}, \gamma_{1}^{\prime}\right) \ldots\left(a_{n}, \gamma_{n}^{\prime}\right)$ that satisfy, for every $a \in \Sigma$ and $1 \leq i \leq n$,

- if $\gamma_{i}\left(x_{a}\right) \leq K$ is an integer then $\gamma_{i}\left(x_{a}\right)-1<\gamma_{i}^{\prime}\left(x_{a}\right)<\gamma_{i}\left(x_{a}\right)+1$, and

- if $\gamma_{i}\left(x_{a}\right) \leq K$ is non-integer or $\gamma_{i}\left(x_{a}\right)>K$ then $\gamma_{i}^{\prime}\left(x_{a}\right) \approx_{K} \gamma_{i}\left(x_{a}\right)$.

We can then define a "closest" adjacent pair as a critical pair, used to infer guards in the tree, by the following definition.

Definition 5.4 Let $w_{g}$ be a guarded word. A critical pair in $w_{g}$ is an adjacent pair $\left(w_{c}, w_{c}^{\prime}\right)$ of clocked words in $w_{g}$ which are distinguished by Obs, such that there is no other adjacent pair $\left(u_{c}, u_{c}^{\prime}\right)$ of clocked words in $w_{g}$ and in neighbourhood $\left(w_{c}\right)$, which are distinguished by Obs, such that $\operatorname{diff}\left(u_{c}, u_{c}^{\prime}\right) \subset$ $\operatorname{diff}\left(w_{c}, w_{c}^{\prime}\right)$.

Intuitively, a critical pair is a pair of clocked words which are distinguished by Obs, and are "as adjacent as possible".

Example 5.5 Let $w_{g}=\left(a, x_{a} \geq 1 \wedge x_{a}<2\right)\left(b, x_{a} \leq 2 \wedge x_{b} \geq 3\right)$, and let the pair $\left(w_{c}, w_{c}^{\prime}\right)$ be distinguished by $O b s$, where

$$
\begin{aligned}
& w_{c}=\left(a,\left(\begin{array}{l}
x_{a} \rightarrow 1.0 \\
x_{b} \rightarrow 1.0
\end{array}\right)\right)\left(b,\left(\begin{array}{c}
x_{a} \rightarrow 2.0 \\
x_{b} \rightarrow 3.0
\end{array}\right)\right) \text { and } \\
& w_{c}^{\prime}=\left(a,\left(\begin{array}{l}
x_{a} \rightarrow 1.5 \\
x_{b} \rightarrow 1.5
\end{array}\right)\right)\left(b,\left(\begin{array}{c}
x_{a} \rightarrow 1.5 \\
x_{b} \rightarrow 3.0
\end{array}\right)\right)
\end{aligned}
$$

Here, $\operatorname{diff}\left(w_{c}, w_{c}^{\prime}\right)=\left\{\left(1, x_{a}, 1, \leq\right),\left(1, x_{b}, 1, \leq\right),\left(2, x_{a}, 2, \geq\right)\right\}$. Since $\left|\operatorname{diff}\left(w_{c}, w_{c}^{\prime}\right)\right|>$ 1 , we search for clocked words in neighbourhood $\left(w_{c}\right)$ and $w_{g}$ which are different from $w_{c}$ and $w_{c}^{\prime}$. Assume that we find

$$
\begin{aligned}
& u_{c}=\left(a,\left(\begin{array}{l}
x_{a} \rightarrow 1.6 \\
x_{b} \rightarrow 1.6
\end{array}\right)\right)\left(b,\left(\begin{array}{l}
x_{a} \rightarrow 2.0 \\
x_{b} \rightarrow 3.6
\end{array}\right)\right) \text { and } \\
& u_{c}^{\prime}=\left(a,\left(\begin{array}{l}
x_{a} \rightarrow 1.6 \\
x_{b} \rightarrow 1.6
\end{array}\right)\right)\left(b,\left(\begin{array}{l}
x_{a} \rightarrow 1.5 \\
x_{b} \rightarrow 3.1
\end{array}\right)\right)
\end{aligned}
$$

Note that any other $v_{c} \vDash w_{g}$ is such that either $v_{c} \approx_{K} w_{c}$ or $v_{c} \approx_{K} w_{c}^{\prime}$ or $v_{c} \approx_{K} u_{c}$, or $v_{c} \approx_{K} u_{c}^{\prime}$. Note that the pairs $\left(w_{c}, w_{c}^{\prime}\right),\left(u_{c}, u_{c}^{\prime}\right),\left(w_{c}, u_{c}\right),\left(w_{c}^{\prime}, u_{c}^{\prime}\right)$, and $\left(w_{c}, u_{c}^{\prime}\right)$ are adjacent pairs, but $\left(w_{c}^{\prime}, u_{c}\right)$ is not an adjacent pair. Now, if $\operatorname{Obs}\left(u_{c}\right) \neq \operatorname{Obs}\left(u_{c}^{\prime}\right)$ then $\operatorname{diff}\left(u_{c}, u_{c}^{\prime}\right)=\left\{\left(2, x_{a}, 2, \geq\right)\right\}$, hence $\left(u_{c}, u_{c}^{\prime}\right)$ is a critical pair in $w_{g}$. If $\operatorname{Obs}\left(u_{c}\right)=\operatorname{Obs}\left(u_{c}^{\prime}\right)$, then, on the one hand, if $\operatorname{Obs}\left(u_{c}\right)=$ $\operatorname{Obs}\left(w_{c}^{\prime}\right)$ (implying $\operatorname{Obs}\left(w_{c}\right) \neq O b s\left(u_{c}\right)$ and $O b s\left(w_{c}\right) \neq O b s\left(u_{c}^{\prime}\right)$ ) it follows from $\operatorname{diff}\left(w_{c}, u_{c}\right) \subset \operatorname{diff}\left(w_{c}, u_{c}^{\prime}\right)$ and $\left|\operatorname{diff}\left(w_{c}, w_{c}^{\prime}\right)\right|=\left|\operatorname{diff}\left(w_{c}, u_{c}\right)\right|$ that $\left(w_{c}, w_{c}^{\prime}\right)$ and $\left(w_{c}, u_{c}\right)$ are critical pairs in $w_{g}$. On the other hand, if $\operatorname{Obs}\left(u_{c}\right)=\operatorname{Obs}\left(u_{c}^{\prime}\right)$ and $\operatorname{Obs}\left(u_{c}\right)=\operatorname{Obs}\left(w_{c}\right)$, we obtain (observing that $\operatorname{diff}\left(w_{c}^{\prime}, u_{c}^{\prime}\right) \not \subset \operatorname{diff}\left(w_{c}, w_{c}^{\prime}\right)$ ) that $\left(w_{c}, w_{c}^{\prime}\right)$ and $\left(w_{c}^{\prime}, u_{c}^{\prime}\right)$ are critical pairs in $w_{g}$. 


\subsection{Separating guards}

The point of a critical pair is that it allows a natural construction of a guard that splits an inconsistent node or some of its ancestors, as described by the following definition.

Definition 5.6 Let $\left(w_{c}, w_{c}^{\prime}\right)$ be a critical pair in $w_{g}$, An atomic guard $x_{a} \leq k$ is a separating guard at position $i$ inferred from $\left(w_{c}, w_{c}^{\prime}\right)$ if

- $\left(i, x_{a}, k, \leq\right) \in \operatorname{diff}\left(w_{c}, w_{c}^{\prime}\right)$ and

- there is no $\left(j, x_{b}, k^{\prime}, \geq\right)$ or $\left(j, x_{b}, k^{\prime}, \leq\right)$ in $\operatorname{diff}\left(w_{c}, w_{c}^{\prime}\right)$ such that

$-j<i$, or

$-j=i$ and $k^{\prime}<k$.

Similarly, from the dual case the separating guard $x_{a} \geq k$ can be inferred.

The definition states that if $\operatorname{diff}\left(w_{c}, w_{c}^{\prime}\right)$ has several elements, then priority is given to the guard at the earliest possible position, and if there is still a choice to resolve it in favour of the smallest constant. A reason for choosing the earliest difference is that such a split still remains in the tree, even if later a split is introduced into the tree which splits one of its ancestors; thus splits are never "undone".

In Example 5.2, we have $\operatorname{diff}\left(w_{c}, w_{c}^{\prime}\right)=\left\{\left(2, x_{b}, 1, \leq\right)\right\}$, whence $\left(w_{c}, w_{c}^{\prime}\right)$ is a critical pair and the separating guard $x_{b} \leq 1$ at position 2 is inferred from it. In Example 5.3, $\operatorname{diff}\left(w_{c}, w_{c}^{\prime}\right)=\left\{\left(1, x_{a}, 1, \geq\right),\left(1, x_{b}, 1, \geq\right)\right\}$. Since $x_{a}$ and $x_{b}$ have the same value at the first position, there are no closer adjacent pairs in the neighborhood of $w_{c}$. Hence $\left(w_{c}, w_{c}^{\prime}\right)$ is critical pair, and there are two possible separating guards, $x_{a} \geq 1$ and $x_{b} \geq 1$ at position 1 . Definition 5.6 can still not give a priority between the two guards, so in this case both $x_{a} \geq 1$ and $x_{b} \geq 1$ are separating guards at position 1 inferred from the critical pair. In cases like this, we have to choose one of the inequalities as a guard in the tree. This choice can influence the size of the inferred automaton, as illustrated in Section 8.

Definition 5.6 describes how to create guards from critical pairs. Since our algorithm attempts to find precisely the guards that occur in $\mathcal{A}$, the automaton to be learned, we require that any guard be introduced only "by need", meaning that it is inferred from a critical pair. This is formalized as the property of being well-guarded.

Definition 5.7 An observation structure $\langle O b s, \mathcal{N}\rangle$ is well-guarded if for each conjunct $g_{i}$ that occurs in the last guard of some node $u_{g}\left(a, g_{1} \wedge \ldots \wedge g_{k}\right)$ in $\mathcal{N}$ there is a critical pair $\left(w_{c}, w_{c}^{\prime}\right)$ which passes $u_{g}$, from which $g_{i}$, or its negation, is inferred at position $i$, where $i=\left|u_{g}\right|+1$.

Intuitively, well-guardedness requires that each conjunct in a guard of the tree be motivated by a critical pair. 


\subsection{Finding Critical Pairs}

In this subsection, we describe how to find a critical pair, when the timed decision tree contains an inconsistent node $w_{g}$, so that $w_{g}$ or one of its ancestors can be split to resolve the inconsistency. The search procedure is an adaptation of binary search, and described in the proof of the following theorem.

Theorem 5.8 Let $\langle O b s, \mathcal{N}\rangle$ be an observation structure. If there is an inconsistent node $w_{g} \in \mathcal{N}$, then a critical pair in $w_{g}$ can be found by $O(m|\Sigma| \log K+$ $\left.3^{m|\Sigma|}\right)$ membership queries, where $m$ is the length of $w_{g}$.

Proof. Assume that the node $w_{g}$ is inconsistent, i.e., there exist two clocked words $w_{c}$ and $w_{c}^{\prime}$, such that

- $w_{c} \vDash w_{g}$ and $w_{c}^{\prime} \vDash w_{g}$,

- $\operatorname{Obs}\left(w_{c}\right) \neq \operatorname{Obs}\left(w_{c}^{\prime}\right)$.

That is, $w_{c}$ and $w_{c}^{\prime}$ lead to the same node, one is accepted and one is rejected. Our goal is to find a critical pair in $w_{g}$.

Our first step is to construct two adjacent clocked words $u_{c}$ and $u_{c}^{\prime}$ leading to $w_{g}$ such that one is accepted and one is rejected. We will use binary search on clocked words. First we define the notion of convex combination of clocked words. For two clocked words $v_{c}=\left(a_{1}, \gamma_{1}\right) \ldots\left(a_{n}, \gamma_{m}\right)$ and $v_{c}^{\prime}=\left(a_{1}, \gamma_{1}^{\prime}\right) \ldots\left(a_{n}, \gamma_{m}^{\prime}\right)$ and two real numbers $\lambda_{1}, \lambda_{2} \in \mathbb{R}^{\geq 0}$ with $\lambda_{1}+\lambda_{2}=1$, define $\lambda_{1} v_{c}+\lambda_{2} v_{c}^{\prime}$ to be the clocked word $\left(a_{1}, \gamma_{1}^{\prime \prime}\right) \ldots\left(a_{n}, \gamma_{m}^{\prime \prime}\right)$ where $\gamma_{i}^{\prime \prime}\left(x_{a}\right)=$ $\lambda_{1} \gamma_{i}\left(x_{a}\right)+\lambda_{2} \gamma_{i}^{\prime}\left(x_{a}\right)$ for all $1 \leq i \leq m$ and $a \in \Sigma$. The binary search algorithm manipulates two clocked words $u_{c}, u_{c}^{\prime}$ that will be moved closer and closer to each other. Initially, $u_{c}=w_{c}$ and $u_{c}^{\prime}=w_{c}^{\prime}$. In each iteration we construct $u_{c}^{\prime \prime}=0.5 u_{c}+0.5 u_{c}^{\prime}$. If $u_{c}^{\prime \prime} \not_{K} u_{c}$ and $u_{c}^{\prime \prime} \not_{K} u_{c}^{\prime}$ a membership query for $u_{c}^{\prime \prime}$ is performed. If $O b s\left(u_{c}\right) \neq O b s\left(u_{c}^{\prime \prime}\right)$ we set $u_{c}^{\prime}$ to the value of $u_{c}^{\prime \prime}$, otherwise we set $u_{c}$ to the value of $u_{c}^{\prime \prime}$. We continue the binary search either until $\gamma_{i}\left(x_{a}\right)>K$ and $\gamma_{i}^{\prime}\left(x_{a}\right)>K$ or until $-1<\gamma_{i}\left(x_{a}\right)-\gamma_{i}^{\prime}\left(x_{a}\right)<1$ for all $1 \leq i \leq m$ and $a \in \Sigma$ where $\left(a_{i}, \gamma_{i}\right)$ is the $i$ th pair in $u_{c}$ and $\left(a_{i}, \gamma_{i}^{\prime}\right)$ is the $i$ th pair in the $u_{c}^{\prime}$.

Assume that there are $i$ and $a$ such that the difference between values of clock $x_{a}$ at position $i$ in $w_{c}$ and $w_{c}^{\prime}$ is much bigger than $K$. Assume that there are $j$ and $b$ such that the difference between values of clock $x_{b}$ at position $j$ in $w_{c}$ and $w_{c}^{\prime}$ is smaller than $K$. Then after $\lceil\log K\rceil$ membership queries binary search construct $u_{c}$ and $u_{c}^{\prime}$ such that the values of clock $x_{b}$ at position $j$ in $u_{c}$ and $u_{c}^{\prime}$ are close to each other, but difference between values of clock $x_{a}$ at position $i$ in $u_{c}$ and $u_{c}^{\prime}$ can be still bigger than $K$. To make values of clock $x_{a}$ at position $i$ close to each other we also need at most $\lceil\log K\rceil$ since some clocked words $u_{c}^{\prime \prime}$ which binary search constructs will be such that $u_{c}^{\prime \prime} \approx_{K} u_{c}$ or $u_{c}^{\prime \prime} \approx_{K} u_{c}^{\prime}$ and we do not need to perform a membership query for $u_{c}^{\prime \prime}$. It follows that for every $1 \leq i \leq m$ and $a \in \Sigma$ at most $\lceil\log K\rceil$ membership queries are performed. Then binary search performs at most $m|\Sigma|\lceil\log K\rceil$ membership queries. 
Intuitively $u_{c}$ and $u_{c}^{\prime}$ are "close", but they need not be adjacent. There can be $i, b$ and $k \leq K, k \in \mathbb{N}$ such that

$$
\gamma_{i}\left(x_{b}\right)>k \text { and } \gamma_{i}^{\prime}\left(x_{b}\right)<k
$$

Then we construct the clocked word $u_{c}^{\prime \prime}$ as a convex combination of $u_{c}$ and $u_{c}^{\prime}$ such that $\left(a_{i}, \gamma_{i}^{\prime \prime}\right)$ is $i$ th pair in $u_{c}^{\prime \prime}$ and $\gamma_{i}^{\prime \prime}\left(x_{b}\right)=k$. It can be done by setting $u_{c}^{\prime \prime}=\lambda u_{c}+(1-\lambda) u_{c}^{\prime}$, where $\lambda=\left(k-\gamma_{i}^{\prime}\left(x_{b}\right)\right) /\left(\gamma_{i}\left(x_{b}\right)-\gamma_{i}^{\prime}\left(x_{b}\right)\right)$. Then a membership query for $u_{c}^{\prime \prime}$ is performed. If $O b s\left(u_{c}^{\prime \prime}\right) \neq O b s\left(u_{c}\right)$ then we set $u_{c}^{\prime}=u_{c}^{\prime \prime}$ otherwise we set $u_{c}=u_{c}^{\prime \prime}$. We repeat this process for $u_{c}$ and $u_{c}^{\prime}$ until we find $u_{c}$ and $u_{c}^{\prime}$ such that for every $i$ and $b$ there is a $k \leq K$ such that

- $\gamma_{i}\left(x_{b}\right) \approx_{K} \gamma_{i}^{\prime}\left(x_{b}\right)$, or

- $\gamma_{i}\left(x_{b}\right)=k$ and $k-1<\gamma_{i}^{\prime}\left(x_{b}\right)<k+1$, or

- $\gamma_{i}^{\prime}\left(x_{b}\right)=k$ and $k-1<\gamma_{i}\left(x_{b}\right)<k+1$.

The number of membership queries performed at this step is at most $m|\Sigma|$.

It can be that $u_{c}$ and $u_{c}^{\prime}$ are still not adjacent, since there are $i, j, a, b$ and $k \leq K, k^{\prime} \leq K$ such that $\gamma_{i}\left(x_{b}\right)=k, \gamma_{i}^{\prime}\left(x_{b}\right) \neq k, \gamma_{j}\left(x_{a}\right) \neq k^{\prime}$ and $\gamma_{j}^{\prime}\left(x_{a}\right)=k^{\prime}$. We can solve this problem by setting $u_{c}^{\prime \prime}=0.5 u_{c}+0.5 u_{c}^{\prime}$. As result we get that $u_{c}^{\prime \prime}$ is adjacent to $u_{c}$ and $u_{c}^{\prime}$. Then we perform membership query for $u_{c}^{\prime \prime}$. Assume $O b s\left(u_{c}\right) \neq O b s\left(u_{c}^{\prime \prime}\right)$ and set $u_{c}^{\prime}=u_{c}^{\prime \prime}$.

Our second step is, starting with $u_{c}$ and $u_{c}^{\prime}$, to construct a critical pair. The critical pair can be found in neighbourhood $\left(u_{c}\right)$ by performing at most $3^{m|\Sigma|}$ membership queries.

Then the number of membership queries that the algorithm performs is $O\left(m|\Sigma| \log K+3^{m|\Sigma|}\right)$.

\subsection{Restructuring a Timed Decision Tree}

In this subsection, we describe our procedure for restructuring a timed decision tree to remove an inconsistent node, after a new critical pair has been found to resolve the inconsistency. The restructuring will affect the subtree rooted at the node which is split by the guard inferred from the critical pair. Thus, the restructuring procedure should produce a new subtree which is well-guarded in the sense of Definition 5.7, and so that for all critical pairs $\left(w_{c}, w_{c}^{\prime}\right)$, where both $w_{c}$ and $w_{c}^{\prime}$ pass the root of the reconstructed subtree, the clocked words $w_{c}$ and $w_{c}^{\prime}$ lead to different nodes.

Our procedure is represented by the function reconstruct_subtree $\left(u_{g}\right)$, presented in Algorithm 1, which returns a restructured subtree rooted at $u_{g}$. It invokes the function find_guards to generate guards on the edges that lead to children of $u_{g}$. The function find_guards infers atomic guards from the relevant critical pairs, and organizes them into a set of conjunctions. In order to guarantee that splits are never "undone", we consider critical pairs in the same ordering as they were added to $\operatorname{Dom}(\mathrm{Obs})$. For each generated child of $u_{g}$, 


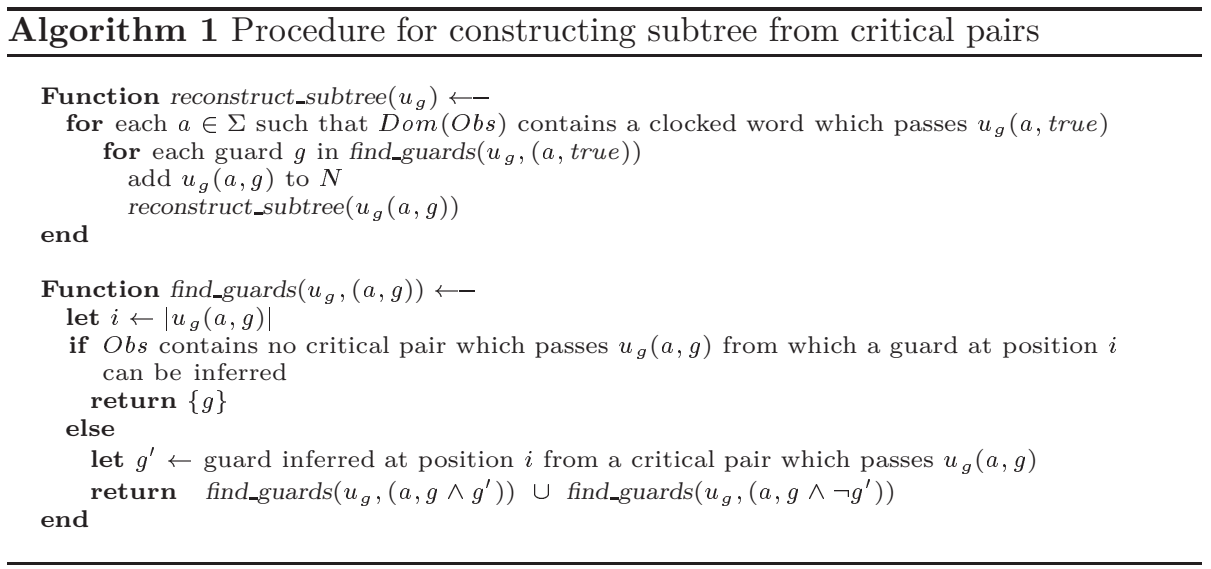

reconstruct_subtree $\left(u_{g}\right)$ invokes itself recursively as long as there are relevant observations in $\mathrm{Obs}$.

That reconstruct_subtree performs its intended function is stated by the following proposition.

Proposition 5.9 Given a partial mapping Obs and a guarded word $u_{g}$, the function reconstruct_subtree $\left(u_{g}\right)$ produces a well-guarded subtree rooted at $u_{g}$, such that for all critical pairs $\left(w_{c}, w_{c}^{\prime}\right)$, where both $w_{c}$ and $w_{c}^{\prime}$ pass $u_{g}$, the clocked words $w_{c}$ and $w_{c}^{\prime}$ lead to different nodes.

In particular, reconstruct_subtree $(\lambda)$ constructs a timed decision tree $\mathcal{N}$ so that $\langle O b s, \mathcal{N}\rangle$ is well-guarded, and so that no critical pair is the cause of an inconsistency.

Proof. Well-guardedness follows by observing that find_guards only infers atomic guards from critical pairs. The second condition follows by noting that find_guards returns only when there are no critical pairs that remain to be separated.

We can use the function reconstruct_subtree $\left(u_{g}\right)$ to remove an inconsistent node as follows. Assume that to the observation structure $\langle O b s, \mathcal{N}\rangle$ we have added a critical pair in $w_{g}$ from which the guard $g^{\prime}$ is inferred at position $i$. Let $u_{g}(a, g)$ be the prefix of length $i$ of $w_{g}$. We then remove the subtree rooted at $u_{g}(a, g)$ and replace it by two new subtrees, one rooted at $u_{g}\left(a, g \wedge g^{\prime}\right)$, and the other rooted at $u_{g}\left(a, g \wedge \neg g^{\prime}\right)$. The first subtree is constructed by adding $u_{g}\left(a, g \wedge g^{\prime}\right)$ and then invoking reconstruct_subtree $\left(u_{g}\left(a, g \wedge g^{\prime}\right)\right)$, and the second by adding $u_{g}\left(a, g \wedge \neg g^{\prime}\right)$ and then invoking reconstruct_subtree $\left(u_{g}\left(a, g \wedge \neg g^{\prime}\right)\right)$.

If, after reconstruction, the tree is still inconsistent (note that the reconstruction does not guarantee absence of inconsistent nodes for which there is no critical pair), then we resolve it by again asking more membership queries in order to find a critical pair and thereafter again reconstruct the tree to remove the inconsistent node. This process can be repeated many times, but the num- 


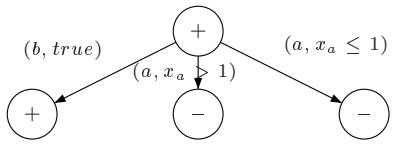

(a)

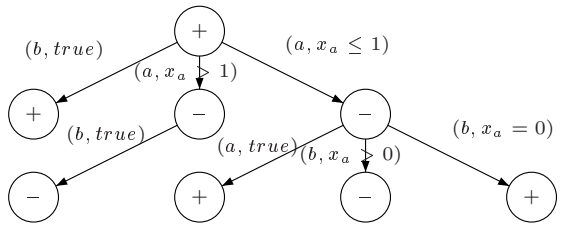

(b)

Figure 2: Introducing a split into the tree

ber of repetitions is bounded, since there is a bound on the number of possible guarded words of a given depth.

Example 5.10 We show an example of how a split is introduced in the tree. Recall the observation structure $\langle O b s, \mathcal{N}\rangle$ shown in Figure 1 . The node $(a$, true $)\left(b, x_{a}=\right.$ 0 ) is inconsistent. In order to resolve the inconsistency we need to find a critical pair. Suppose that the critical pair is

$$
\begin{aligned}
& u_{c}=\left(a,\left(\begin{array}{c}
x_{a} \rightarrow 1.0 \\
x_{b} \rightarrow 1.0
\end{array}\right)\right)\left(b,\left(\begin{array}{c}
x_{a} \rightarrow 0.0 \\
x_{b} \rightarrow 1.0
\end{array}\right)\right), \\
& u_{c}^{\prime}=\left(a,\left(\begin{array}{c}
x_{a} \rightarrow 1.5 \\
x_{b} \rightarrow 1.5
\end{array}\right)\right)\left(b,\left(\begin{array}{c}
x_{a} \rightarrow 0.0 \\
x_{b} \rightarrow 1.5
\end{array}\right)\right)
\end{aligned}
$$

We add $u_{c}, u_{c}^{\prime}$ to $\operatorname{Dom}(\mathrm{Obs})$. There are two separating guards at position 1 which can be inferred from it. We choose the guard $x_{a} \leq 1$. Then we remove (a,true) and all its descendants from $\mathcal{N}$ and add $\left(a, x_{a} \leq 1\right)$ and $\left(a, x_{a}>1\right)$ to $\mathcal{N}$, see Figure $2(\mathrm{a})$.

There is no critical pair which passes $\left(a, x_{a}>1\right)(b$, true $)$ or $\left(a, x_{a} \leq 1\right)(a$, true $)$. Since $(a, 1.5)(b, 1.5) \in \operatorname{Dom}($ Obs $)$ passes $\left(a, x_{a}>1\right)(b$, true $)$ and $(a, 0)(a, 0) \in$ $\operatorname{Dom}($ Obs $)$ passes $\left(a, x_{a} \leq 1\right)(a$, true $)$ then we add $\left(a, x_{a}>1\right)(b$, true $)$ and $\left(a, x_{a} \leq 1\right)(a$, true $)$ to $\mathcal{N}$.

There is a critical pair

$$
\begin{aligned}
& w_{c}=\left(a,\left(\begin{array}{c}
x_{a} \rightarrow 0.5 \\
x_{b} \rightarrow 0.5
\end{array}\right)\right)\left(b,\left(\begin{array}{c}
x_{a} \rightarrow 0.0 \\
x_{b} \rightarrow 0.5
\end{array}\right)\right), \\
& w_{c}^{\prime}=\left(a,\left(\begin{array}{c}
x_{a} \rightarrow 0.5 \\
x_{b} \rightarrow 0.5
\end{array}\right)\right)\left(b,\left(\begin{array}{c}
x_{a} \rightarrow 0.1 \\
x_{b} \rightarrow 0.6
\end{array}\right)\right)
\end{aligned}
$$

in $\operatorname{Dom}(\mathrm{Obs})$ which passes $\left(a, x_{a} \leq 1\right)(b$, true $)$. Since the separating clock guard $x_{a}=0$ at position 2 is inferred from $\left(w_{c}, w_{c}^{\prime}\right)$, we add $\left(a, x_{a} \leq 1\right)\left(b, x_{a}=0\right)$ and $\left(a, x_{a} \leq 1\right)\left(b, x_{a}>0\right)$ to $\mathcal{N}$, see Figure $2(\mathrm{~b})$. Thereafter $\langle O b s, \mathcal{N}\rangle$ is a well-formed and consistent observation structure.

\section{Constructing Automata from Observation Struc- tures}

In this section, we describe how to fold a timed decision tree into a hypothesized TDERA, by merging nodes which have "similar" future behavior according to Obs. Since the construction is not trivial, we will first provide some motivation and an illustrating example. 


\subsection{Informal Overview}

As motivation, let us consider the simpler case of untimed automata, which can be regarded as TDERAs in which all guards are true. When performing regular inference for untimed automata, following the principles used in e.g., [Ang87, Gol67, KV94, RS93, BDG97], we can first construct a timed decision tree with all guards being true, which is essentially a mapping from a prefixclosed set $\operatorname{Dom}(\mathrm{Obs})$ of untimed words in $\Sigma^{*}$ to $\{+,-\}$. To fold this mapping into an automaton, we find a prefix-closed subset $U$ of $\operatorname{Dom}(\mathrm{Obs})$, such that for all words $u \in U$ and alphabet symbols $a \in \Sigma$, the extension $u a$ is in $\operatorname{Dom}(O b s)$, and furthermore if $u a$ is not in $U$ then $u a$ can be merged with some word $u^{\prime}$ in $U$. Since we want the resulting automaton to agree with $O b s$, this merging should be done only if the observed future behavior from $u a$ is included in the observed future behavior from $u^{\prime}$, i.e., whenever $u a z \in \operatorname{Dom}(\mathrm{Obs})$ for some suffix $z \in \Sigma^{*}$, then $u^{\prime} z \in \operatorname{Dom}(\mathrm{Obs})$ and $\mathrm{Obs}\left(u^{\prime} z\right)=\operatorname{Obs}(u a z)$.

If in addition we require that for any two words $u, u^{\prime}$ in $U$, there is some suffix $z$ that separates them, i.e., $u z, u^{\prime} z \in \operatorname{Dom}(\mathrm{Obs})$ and $O b s(u z) \neq O b s\left(u^{\prime} z\right)$, then we can prove that the set $U$, which forms the locations of the resulting hypothesized automaton $\mathcal{H}$, will have at most as many members as there are locations in the automaton $\mathcal{A}$ to be learned [Ang87]. Namely, define a mapping $\theta$ from $U$ to locations in $\mathcal{A}$ by $\theta(u)=l$ if the word $u$ takes $\mathcal{A}$ from its initial location to $l$. We note that if $u$ and $u^{\prime}$ are two different words in $U$, then $\theta(u) \neq \theta\left(u^{\prime}\right)$ since $u$ and $u^{\prime}$ are separated by some suffix. Hence $U$ cannot have more words than there are locations of $\mathcal{A}$. This bound on $\mathcal{H}$ can then be used to prove that the inference algorithm terminates [Ang87].

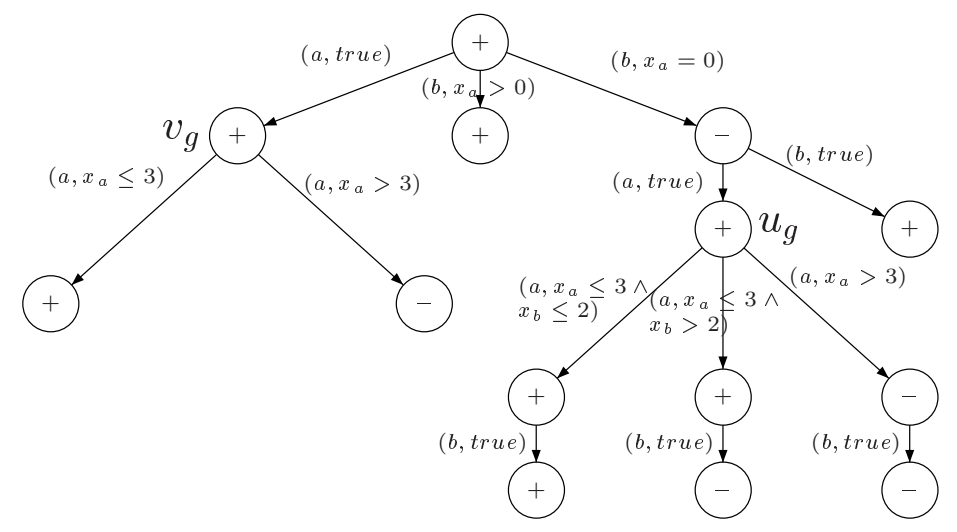

Figure 3: Illustration of inclusion between subtrees

Let us now consider how these principles can be transferred to the inference of TDERAs. A main question is to define the requirements for when a node (i.e., a guarded word) $u(a, g)$ in a timed decision tree can be merged with another node $u^{\prime}$. It is natural to require that the subtree rooted at $u(a, g)$ be "included" in the subtree rooted at $u^{\prime}$. Consider, e.g., the timed decision tree in Figure 3. 
Here, the subtree rooted at the node $v_{g}=(a, t r u e)$ to the left accepts all clocked suffix words of form $(a, \gamma)$ such that $\gamma\left(x_{a}\right) \leq 3$, and rejects all clocked suffix words of form $(a, \gamma)$ such that $\gamma\left(x_{a}\right)>3$. This information is also included in the subtree rooted at the node $u_{g}=\left(b, x_{a}=0\right)(a$, true $)$ to the right, hence we say that the subtree rooted at $v_{g}$ is "included" in the subtree rooted at $u_{g}$, and allow $v_{g}$ to be merged with $u_{g}$ (for clarity, we have omitted edges from $u_{g}$ and $v_{g}$ for the symbol $b$ ). In Theorem 6.5 , we establish that with inclusion between subtrees as the criterion for merging guarantees that the resulting hypothesized automaton $\mathcal{H}$ agrees with $O b s$.

A problem with the above merging requirement is that we cannot imitate the termination proof of the untimed case, in which separating suffixes play a crucial role to establish a bound on the size of constructed automata. The reason is that even if two nodes have different subtrees which cannot be merged, it may not be possible to find a separating suffix. To understand why this is the case, note that each node in the tree has a postcondition which restricts the suffixes of the node. For instance, in Figure 3 the nodes $u_{g}$ and $v_{g}$ have the postcondition $x_{a} \leq x_{b}$, which implies that, e.g., any suffix of form $(a, \gamma)$ which can occur in a membership query must satisfy $\gamma\left(x_{a}\right) \leq \gamma\left(x_{b}\right)$. This shows that in general, two subtrees may disagree on the acceptance of suffixes that cannot occur in queries, implying that their roots cannot be separated by a suffix. A

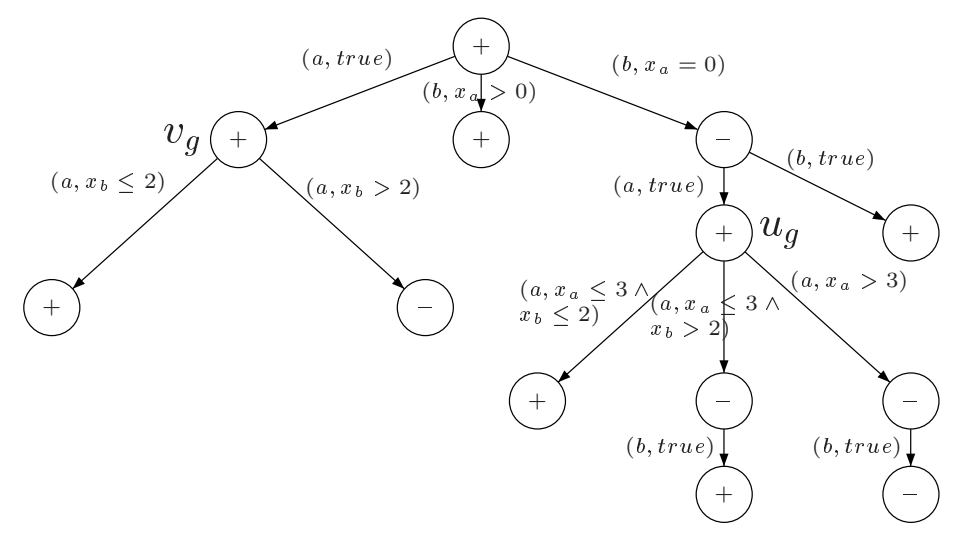

Figure 4: Illustration of alternative merging criterion

concrete example is found in Figure 4 . Here, the subtrees rooted at the nodes $v_{g}$ and $u_{g}$ disagree on the acceptance of, e.g., the clocked suffix word $(a, \gamma)$ where $\gamma\left(x_{a}\right)=4$ and $\gamma\left(x_{b}\right)=2$. However, this suffix cannot occur in a query since it does not satisfy the postcondition $x_{a} \leq x_{b}$ at $v_{g}$ and $u_{g}$.

A way to imitate the termination argument in the untimed case is to define a different criterion for merging nodes, which requires that the two nodes have region-equivalent postconditions, and that their subtrees agree on suffixes that can actually occur in queries. As an illustration, consider the timed decision tree in Figure 4. Here, the subtree rooted at the node $v_{g}$ to the left accepts all 
clocked suffix words of form $(a, \gamma)$ such that $\gamma\left(x_{b}\right) \leq 2$, and rejects all clocked suffix words of form $(a, \gamma)$ such that $\gamma\left(x_{b}\right)>2$. Thus, the node $v_{g}$ cannot be merged with the node $u_{g}$ according to the previous criterion. However, if we restrict attention to suffixes that satisfy the postcondition $x_{a} \leq x_{b}$, then the subtree rooted at $u_{g}$ accepts all clocked suffix words of form $(a, \gamma)$ such that $\gamma\left(x_{b}\right) \leq 2$, and rejects all clocked suffix words of form $(a, \gamma)$ such that $\gamma\left(x_{b}\right)>2$, since any suffix of form $(a, \gamma)$ which satisfies the postcondition $x_{a} \leq x_{b}$ and $\gamma\left(x_{a}\right)>3$ also satisfies $\gamma\left(x_{b}\right)>2$.

With the new merging criterion, we can indeed require that two nodes in $U$ which are not merged and have region-equivalent postconditions, must be separated by some suffix. A drawback of this new relation, however, is that nodes with postconditions which are not region-equivalent cannot be merged. In contrast, the first criterion, based on inclusion between subtrees, allows to merge nodes with postconditions which are not region-equivalent, often resulting in significantly smaller automata. In order to generate automata that are always of bounded size and often small, we therefore present a restructuring operation on trees, called "copying", with the goal that nodes which can be merged by the second criterion can also be merged by the first. Intuitively, the copying operation copies the structure of one subtree to that of another. For instance, in Figure 4 , after copying the structure of the subtree rooted at $u_{g}$ to the subtree rooted at $v_{g}$, we obtain the tree in Figure 5. After this operation, the subtree rooted at $v_{g}$ is included in the subtree rooted at $u_{g}$. In general, the copying

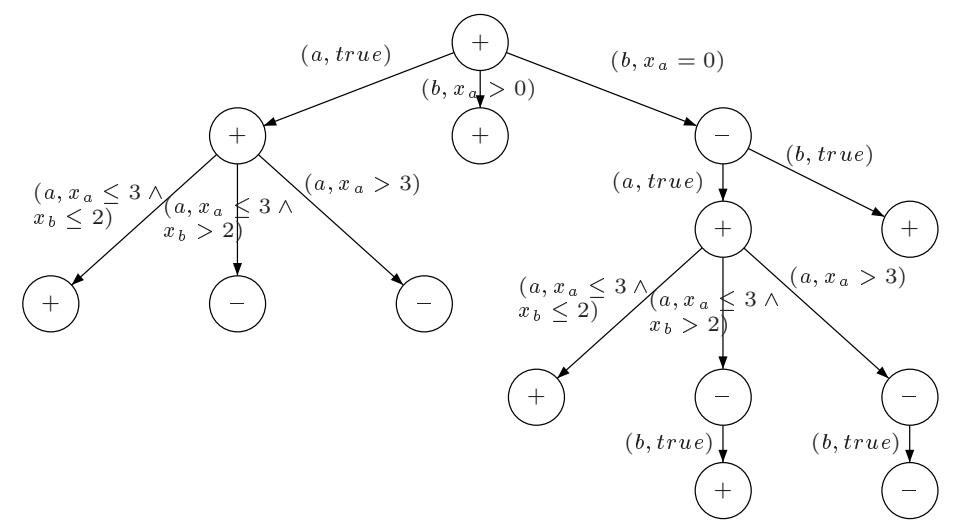

Figure 5: Result after copying operation

of nodes is nontrivial since it is recursive. For instance, it could be that a descendant of $u_{g}$ has its structure copied from some other node.

To summarize, our procedure for folding a timed decision tree into a TDERA consists in first finding a prefix $V$ of nodes, which allows an automaton to be formed by merging nodes according to the second criterion, so that we can prove a bound on the size of $V$. Thereafter, we perform the copying operation, and try to obtain a smaller TDERA by merging nodes according to the first criterion. 
A precondition for this procedure is that the timed decision tree is well-formed and consistent. The bound on $V$ further requires that sufficiently many queries are performed to have separating suffixes wherever needed. We call this latter property region-consistency.

This section is organized as follows. After presentation of a general operation for folding a tree into an automaton in the next section, in Section 6.3, we present the first merging criterion, based on "inclusion" between subtrees. In Section 6.4, we present the second construction, based on suffixes of observations. Section 6.5 describes the copying operation. In Section 7, we put all the pieces together by presenting the overall algorithm, and finally, in Section 7.1, we prove termination and give complexity bounds.

\subsection{Preliminaries}

Let $\mathcal{N}$ be a timed decision tree, and let $u_{g}, v_{g} \in \mathcal{N}$. Define untimesuff $\mathcal{N}_{\mathcal{N}}\left(u_{g}\right)$ as the set of (untimed) words $z$ such that $\mathcal{N}$ contains a descendant $u_{g} z_{g}$ of $u_{g}$ with $z=$ untime $\left(z_{g}\right)$. Let height $\left(u_{g}, \mathcal{N}\right)$ denote the height of the subtree rooted at $u_{g}$ in $\mathcal{N}$. If $\leq$ is a preorder on nodes in $\mathcal{N}$, we use $v_{g}<u_{g}$ to denote that $v_{g} \leq u_{g}$ and $u_{g} \leq v_{g}$. We say that $\leq$ is height-monotone if $v_{g} \leq u_{g}$ implies height $\left(v_{g}, \mathcal{N}\right) \leq \operatorname{height}\left(u_{g}, \mathcal{N}\right)$. For a set $U$ of nodes of $\mathcal{N}$, let $v_{g} \leq U$ denote that $v_{g} \leq u_{g}$ for some $u_{g} \in U$, and let $\operatorname{succ}(U)$ denote the set of children of nodes in $U$ which are not themselves in $U$.

Definition 6.1 Let $\mathcal{N}$ be a timed decision tree, and let $\leq$ be a preorder on nodes in $\mathcal{N}$, A prefix-closed subset $U$ of $\mathcal{N}$ is called

- complete if for all $u_{g} \in U$ and $a \in \Sigma$ there is some node $u_{g}(a, g) \in \mathcal{N}$,

- $\leq$-closed if $v_{g} \leq U$ for all $v_{g} \in \operatorname{succ}(U)$,

- $\leq$-unique if $U$ does not contain two nodes $u_{g}, u_{g}^{\prime}$ with $u_{g} \leq u_{g}^{\prime}$.

Intuitively, if a prefix-closed subset $U$ of $\mathcal{N}$ is complete and $\leq$-closed, then $\mathcal{N}$ can be folded into a TDERA by merging each node $v_{g}$ in $\operatorname{succ}(U)$ with some $u_{g} \in U$ such that $v_{g} \leq u_{g}$. The property of $\leq$-uniqueness will be used to bound the size of the resulting TDERA.

In the following subsections, we will present several constructions of an automaton from a timed decision tree, in which the locations of the automaton are a prefix-closed set of nodes of the tree. The following lemma gives conditions under which the existence of such a prefix-closed set of nodes can be guaranteed.

Lemma 6.2 Let $\leq$ be a height-monotone preorder on nodes in $\mathcal{N}$. Then there exists $a \leq$-closed and $\leq$-unique prefix-closed subset of $\mathcal{N}$.

Proof. Construct a set $U$ of nodes of $\mathcal{N}$ by starting from the set $\{\lambda\}$ containing only the root, and repeatedly adding to $U$ some successor $v_{g}$ of a node already in $U$ which satisfies 
- $v_{g} \not \leq U$, and

- if $\mathcal{N}$ contains a node $u_{g}^{\prime}$ with $v_{g}<u_{g}^{\prime}$, then $u_{g}^{\prime}$ has an ancestor $v_{g}^{\prime}$ in $\operatorname{succ}(U)$ with $v_{g}^{\prime} \leq U$.

We claim that when the addition of nodes to $U$ cannot be continued, then $U$ is prefix-closed, $\leq$-closed and $\leq$-unique.

- By construction, $U$ is prefix-closed.

- To see that $U$ is $\leq$-unique, note that when a node $v_{g}$ is added to $U$, then $U$ does not contain any $u_{g}$ with $v_{g} \leq u_{g}$; furthermore, if $U$ would contain some $u_{g}$ with $v_{g}>u_{g}$, then at the time when $u_{g}$ was added, $v_{g}$ would have an ancestor $u_{g}^{\prime}$ in $\operatorname{succ}(U)$ with $u_{g}^{\prime} \leq U$, implying that $u_{g}^{\prime}$ and hence $v_{g}$ will never be added to $U$.

- Finally, if $U$ is not $\leq$-closed at termination, then there exist one or several nodes $v_{g}$ in $\operatorname{succ}(U)$ such that $v_{g} \not U$. Each such node $v_{g}$ satisfies (since it cannot be added) $v_{g}<u_{g}^{\prime}$ for some node $u_{g}^{\prime}$ in $\mathcal{N} \backslash U$, which has a unique ancestor $v_{g}^{\prime}$ in $\operatorname{succ}(U)$ with $v_{g}^{\prime} \not Z U$. The node $v_{g}^{\prime}$ in its turn satisfies (for the same reason) $v_{g}^{\prime}<u_{g}^{\prime \prime}$ for some node $u_{g}^{\prime \prime}$ in $\mathcal{N} \backslash U$, which must have an ancestor $v_{g}^{\prime \prime}$ in $\operatorname{succ}(U)$ with $v_{g}^{\prime \prime} \not U U$. Continuing in this way, we must eventually construct a sequence of nodes $v_{g}^{0} v_{g}^{1} v_{g}^{2} \cdots v_{g}^{n}$ with $v_{g}=v_{g}^{0}$ which has a cycle, i.e., $v_{g}^{k}=v_{g}^{n}$ for some $k<n$. However, if the strict ancestor relation is used somewhere in the cycle from $v_{g}^{k}$ to $v_{g}^{n}$, then we have height $\left(v_{g}^{k}, \mathcal{N}\right)<\operatorname{height}\left(v_{g}^{n}, \mathcal{N}\right)$ which contradicts $v_{g}^{k}=v_{g}^{n}$, and if the strict ancestor relation is never used in the cycle from $v_{g}^{k}$ to $v_{g}^{n}$, then we have $v_{g}^{k}<v_{g}^{k+1}<\cdots<v_{g}^{n}$, which also contradicts $v_{g}^{k}=v_{g}^{n}$.

We observe that the proof also provides a natural method for constructing a $\leq$-closed and $\leq$-unique prefix-closed subset of $\mathcal{N}$.

Given a certain prefix $U$ of nodes of a timed decision tree $\mathcal{N}$, we can construct an ERA as follows.

Definition 6.3 Let $\langle O b s, \mathcal{N}\rangle$ be a consistent observation structure, let $\leq$ be a height-monotone preorder on nodes in $\mathcal{N}$, and let $U$ be a $\leq$-unique, $\leq$-closed, and prefix-closed subset of $\mathcal{N}$. Then a $U_{\leq}$-merging of $\langle\overline{O b s}, \mathcal{N}\rangle$ is an ERA $\left\langle U,\{\lambda\}, L^{f}, E\right\rangle$, such that

- $L^{f}=\left\{u_{g} \in U: \operatorname{Obs}\left(u_{g}\right)=+\right\}$, and

- for each node $u_{g}(a, g) \in \mathcal{N}$ with $u_{g} \in U$ there is exactly one edge in $E$ of form $\left(u_{g}, u_{g}^{\prime}, a, g\right) \in E$ with $u_{g}(a, g) \leq u_{g}^{\prime}$.

We observe that if, in addition, $U$ is complete, then $\langle$ Obs, $\mathcal{N}\rangle$ is a TDERA.

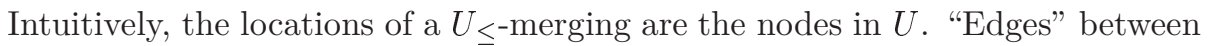
nodes in $U$ are also edges of the automaton, since (by $\leq$-uniqueness of $U$ ) for an edge of form $\left(u_{g}, u_{g}^{\prime}, a, g\right)$ the node $u_{g}^{\prime}$ must be $u_{g}(a, g)$ if $u_{g}(a, g) \in U$. In addition, each "edge" from a node $u_{g}$ in $U$ to a node $u_{g}(a, g)$ in $\operatorname{succ}(U)$ becomes an edge in the automaton from $u_{g}$ to a node $u_{g}^{\prime}$ with $u_{g}(a, g) \leq u_{g}^{\prime}$. 


\subsection{Constructing an Agreeing Automaton}

In this section, we specialize the construction of Definition 6.3 by defining a relation, denoted $\leq_{u n}$, between nodes. Intuitively $v_{g} \leq_{u n} u_{g}$ defines the first merging criterion described in Section 6.1, and means that the subtree rooted at node $v_{g}$ is "included" in the subtree rooted at node $u_{g}$, implying that $v_{g}$ can be merged with $u_{g}$. We then prove that by using $\leq_{u n}$ as a criterion for merging nodes, the result is a TDERA which agrees with $O b s$.

Intuitively, when comparing nodes to see whether they can be merged, we compare their overlapping suffixes, since these represent clocked words that pass the nodes. Two guarded words $w_{g}$ and $w_{g}^{\prime}$ are suffix-overlapped if there is a clocked suffix word $w_{c}$ such that $w_{c} \vDash w_{g}$ and $w_{c} \vDash w_{g}^{\prime}$.

Definition 6.4 Let $\langle O b s, \mathcal{N}\rangle$ be a consistent observation structure. A node $v_{g} \in \mathcal{N}$ is unifiable with a node $u_{g} \in \mathcal{N}$, denoted $v_{g} \leq_{u n} u_{g}$, if

- untimesuff, $\left(v_{g}\right) \subseteq$ untimesuff $_{\mathcal{N}}\left(u_{g}\right)$, and

- for any descendants $v_{g} z_{g}$ of $v_{g}$ and $u_{g} z_{g}^{\prime}$ of $u_{g}$ with $O b s\left(v_{g} z_{g}\right) \in\{+,-\}$ such that $z_{g}$ and $z_{g}^{\prime}$ are suffix-overlapped, we have Obs $\left(v_{g} z_{g}\right)=O b s\left(u_{g} z_{g}^{\prime}\right)$.

It follows from Definition 6.4 that $\leq_{u n}$ is height-monotone, transitive, and hence a preorder. Hence by Lemma 6.2 there exists a $\leq_{u n}$-closed and $\leq_{u n}$-unique prefix-closed subset $U$ of $\mathcal{N}$, from which we can construct an $U_{\leq_{u n}}$-merging. The following theorem states that this $U_{\leq_{u n}}$-merging agrees with $\mathrm{Obs}$.

Theorem 6.5 Let $\langle O b s, \mathcal{N}\rangle$ be a consistent observation structure. Let $U$ be a

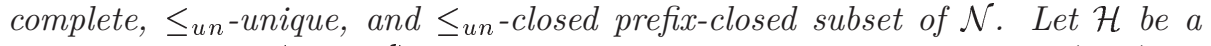
$U_{\leq_{u n}}$-merging of $\langle O b s, \mathcal{N}\rangle$. Then for every clocked word $w_{c} \in \operatorname{Dom}(O b s)$, if Obs $\left(w_{c}\right)=+$ then $w_{c}$ is accepted by $\mathcal{H}$, otherwise $w_{c}$ is rejected.

Proof. Let $w_{c} \in \operatorname{Dom}(\mathrm{Obs})$ and $\operatorname{Obs}\left(w_{c}\right)=+$. Then there is a guarded word $w_{g} \in \mathcal{N}$ with $\operatorname{Obs}\left(w_{g}\right)=+$ such that $w_{c} \vDash w_{g}$. If $w_{g} \in U$ then the lemma trivially holds. If $w_{g} \notin U$, then $w_{g}=u_{g} z_{g}$ for some $u_{g} \in U$ and suffix $z_{g}$ of $w_{g}$. We prove by induction over the length of the guarded word $z_{g}$ that whenever $u_{g} \in U$ and $O b s\left(u_{g} z_{g}\right)=+$ then each clocked suffix word $z_{c}$ such that $z_{c} \vDash z_{g}$ is in $\mathcal{L}_{c}^{+}\left(\mathcal{H}, u_{g}\right)$.

The base case, where $z_{g}=\lambda$, follows trivially as above. Otherwise we can decompose $z_{g}$ as $z_{g}=v_{g} z_{g}^{\prime}$, where $v_{g}$ is nonempty and $u_{g} v_{g} \in \operatorname{succ}(U)$. By Definition 6.3, using that $U$ is $\leq_{u n}$-complete, there is a $u_{g}^{\prime} \in U$ such that $u_{g} v_{g} \leq_{u n} u_{g}^{\prime}$. Let $z_{c}^{\prime}$ be the suffix of $z_{c}$ with $\left|z_{c}^{\prime}\right|=\left|z_{g}^{\prime}\right|$. By Definition 6.4 there is a descendant $u_{g}^{\prime} z_{g}^{\prime \prime}$ of $u_{g}^{\prime}$ such that $z_{c}^{\prime} \vDash z_{g}^{\prime \prime}$, i.e., $z_{g}^{\prime}$ and $z_{g}^{\prime \prime}$ are suffix-overlapped, and $O b s\left(u_{g}^{\prime} z_{g}^{\prime \prime}\right)=+$. By the induction hypothesis (since $z_{g}^{\prime \prime}$ is shorter than $z_{g}$ )

$z_{c}^{\prime}$ is in $\mathcal{L}_{c}^{+}\left(\mathcal{H}, u_{g}^{\prime}\right)$. Hence $z_{c}$ is in $\mathcal{L}_{c}^{+}\left(\mathcal{H}, u_{g}\right)$ (since $u_{g} \stackrel{v_{g}}{\Rightarrow} u_{g}^{\prime} \stackrel{z_{g}^{\prime \prime}}{\Rightarrow} l^{\prime}$ in $\mathcal{H}$ for some accepting location $l^{\prime}$ ).

The case $O b s\left(w_{c}\right)=-$ is analogous. 


\subsection{Constructing Automata of Bounded Size}

The construction in the preceding subsection produces, by Theorem 6.5, a TDERA which agrees with Obs. However, it seems difficult to use this construction to establish a bound the size of the constructed automaton, which is an important step in proving that the Learner eventually infers a TDERA equivalent to $\mathcal{A}$, after a bounded number of membership and equivalence queries. A main reason is that the relation $\leq_{u n}$ compares nodes by the form of their respective subtrees. Since the number of different possible subtrees is unbounded, we can get an unbounded number of incomparable nodes. In this section, we present another relation for comparing nodes, which states that two nodes with region-equivalent postconditions can be merged if they are not separated by some suffix. This will allow to prove a bound on the constructed automata, as described in the informal overview of Section 6.1 .

A precondition for our construction is that the observation structure contains enough queries to satisfy two conditions:

- queries for clocked words that pass some node should, when possible, use the same clocked word as prefix,

- each such prefix should have queries for enough suffixes to make it possible to compare nodes.

To define these conditions, we partition the clocked words that lead to some node $u_{g}$ into equivalence classes, such that two clocked words are equivalent if they can be extended with equivalent suffixes.

Definition 6.6 For two clocked words $u_{c}$ and $u_{c}^{\prime}$, define $u_{c} \simeq_{K} u_{c}^{\prime}$ if for each clocked suffix word $z_{c}$ such that $u_{c} z_{c}$ is a clocked word, there is a clocked suffix word $z_{c}^{\prime}$ with $z_{c} \approx_{K} z_{c}^{\prime}$ such that $u_{c}^{\prime} z_{c}^{\prime}$ is a clocked word, and vice versa.

The following lemma characterizes $\simeq_{K}$ in terms of region equivalence.

Lemma 6.7 Let $u_{c}$ and $u_{c}^{\prime}$ be two clocked words. Then $u_{c} \simeq_{K} u_{c}^{\prime}$ if and only if either $u_{c}=u_{c}^{\prime}=\lambda$ or $\gamma\left[x_{a} \rightarrow 0\right] \sim_{K} \gamma^{\prime}\left[x_{a^{\prime}} \rightarrow 0\right]$, where $(a, \gamma)$ and $\left(a^{\prime}, \gamma^{\prime}\right)$ is the last pair in $u_{c}$ and $u_{c}^{\prime}$, respectively.

Intuitively, $u_{c} \simeq_{K} u_{c}^{\prime}$ if $u_{c}$ and $u_{c}^{\prime}$ lead to the same initial region.

Proof. We first establish the if-direction. Let $u_{c}$ and $u_{c}^{\prime}$ be as in the statement of the lemma, and assume that $\gamma\left[x_{a} \rightarrow 0\right] \sim_{K} \gamma^{\prime}\left[x_{a^{\prime}} \rightarrow 0\right]$, where $(a, \gamma)$ and $\left(a^{\prime}, \gamma^{\prime}\right)$ is the last pair in $u_{c}$ and $u_{c}^{\prime}$, respectively. By Definition 6.6, we must prove that for each clocked suffix word $z_{c}$ such that $u_{c} z_{c}$ is a clocked word, there is a clocked suffix word $z_{c}^{\prime}$ with $z_{c} \approx_{K} z_{c}^{\prime}$ such that $u_{c}^{\prime} z_{c}^{\prime}$ is a clocked word. The case $z_{c}=\lambda$ is trivial. To prove the case $\left|z_{c}\right|=1$, let $(b, \rho)$ be a pair consisting of a symbol $b \in \Sigma$ and clock valuation $\rho$ such that $u_{c}(b, \rho)$ is a clocked word. We will prove the claim that there is a clock valuation $\rho^{\prime}$ with $\rho \approx_{K} \rho^{\prime}$ such that $u_{c}^{\prime}\left(b, \rho^{\prime}\right)$ is a clocked word and $\rho\left[x_{b} \rightarrow 0\right] \sim_{K} \rho^{\prime}\left[x_{b} \rightarrow 0\right]$. We can then use 
this claim to prove the lemma for the general case by induction: simply add one pair, consisting of a symbol and a clock valuation, at a time.

To prove the claim, we must, by the definition of $\sim_{K}$ in Section 2, establish that

A for all $x_{c} \in C_{\Sigma}$, either

- $\rho\left(x_{c}\right)$ and $\rho^{\prime}\left(x_{c}\right)$ are both greater than $K$, or

$-\left\lfloor\rho\left(x_{c}\right)\right\rfloor=\left\lfloor\rho^{\prime}\left(x_{c}\right)\right\rfloor$ and $\operatorname{fract}\left(\rho\left(x_{c}\right)\right)=0$ iff $\operatorname{fract}\left(\rho^{\prime}\left(x_{c}\right)\right)=0$,

and

B for all $x_{c}, x_{c^{\prime}} \in C_{\Sigma}$ where $c, c^{\prime}$ differ from $b$, with $\rho\left(x_{c}\right) \leq K$ and $\rho\left(x_{c^{\prime}}\right) \leq$ $K$

$\operatorname{fract}\left(\rho\left(x_{c}\right)\right) \leq \operatorname{fract}\left(\rho\left(x_{c^{\prime}}\right)\right)$ iff $\operatorname{fract}\left(\rho^{\prime}\left(x_{c}\right)\right) \leq \operatorname{fract}\left(\rho\left(x_{c^{\prime}}\right)\right)$.

The condition on clocked suffix words in Section 2 states that $\rho\left(x_{c}\right)=\rho\left(x_{a}\right)+$ $\gamma\left(x_{c}\right)$ for all $x_{c} \in C_{\Sigma}$ except $x_{a}$. This means that $\rho$ is determined from $\rho\left(x_{a}\right)$, and that we must just find an appropriate value of $\rho^{\prime}\left(x_{a}^{\prime}\right)$ to determine $\rho^{\prime}$. Let $d$ be the symbol in $\Sigma$ such that $\rho\left(x_{d}\right) \leq K$, and such that $\rho\left(x_{d}\right)$ has the least fractional part among all clocks $c$ with $\rho\left(x_{c}\right) \leq K$. Choose a value of $\rho^{\prime}\left(x_{a}^{\prime}\right)$ such that $\rho^{\prime}\left(x_{d}\right) \leq K$, and such that $\rho^{\prime}\left(x_{d}\right)$ has the least fractional part among all clocks $c$ with $\rho^{\prime}\left(x_{c}\right) \leq K$. Then condition A follows from $\gamma\left[x_{a} \rightarrow 0\right] \sim_{K} \gamma^{\prime}\left[x_{a^{\prime}} \rightarrow 0\right]$. Condition B also follows from $\gamma\left[x_{a} \rightarrow 0\right] \sim_{K} \gamma^{\prime}\left[x_{a^{\prime}} \rightarrow 0\right]$, since differences between clock valuations are preserved for all clocks except $x_{b}$.

To prove the only if-direction, assume that $\gamma\left[x_{a} \rightarrow 0\right] \not_{K} \gamma^{\prime}\left[x_{a^{\prime}} \rightarrow 0\right]$. We shall find a pair $(b, \rho)$ consisting of a symbol $b \in \Sigma$ and clock valuation $\rho$ such that $u_{c}(b, \rho)$ is a clocked word, but there is no clock valuation $\rho^{\prime}$ with $\rho \approx_{K} \rho^{\prime}$ such that $u_{c}^{\prime}\left(b, \rho^{\prime}\right)$ is a clocked word or vice versa. If there is some clock $c$ such that $\gamma\left[x_{a} \rightarrow 0\right]\left(x_{c}\right) \not \varkappa_{K} \gamma^{\prime}\left[x_{a^{\prime}} \rightarrow 0\right]\left(x_{c}\right)$, then if $\gamma\left[x_{a} \rightarrow 0\right]\left(x_{c}\right)<\gamma^{\prime}\left[x_{a^{\prime}} \rightarrow 0\right]\left(x_{c}\right)$ we can simply choose $b$ as $c$ and $\rho$ as $\gamma\left[x_{a} \rightarrow 0\right]$ Otherwise, if for instance $\operatorname{fract}\left(\gamma\left[x_{a} \rightarrow 0\right]\left(x_{c}\right)\right)<\operatorname{fract}\left(\gamma\left[x_{a} \rightarrow 0\right]\left(x_{c^{\prime}}\right)\right)$ but $\operatorname{fract}\left(\gamma^{\prime}\left[x_{a^{\prime}} \rightarrow 0\right]\left(x_{c}\right)\right) \geq$ $\operatorname{fract}\left(\gamma\left[x_{a^{\prime}} \rightarrow 0\right]\left(x_{c^{\prime}}\right)\right)$ we can find a constant $k \leq K$ and $\rho$ such that $\rho\left(x_{c}\right)<$ $k<\rho\left(x_{c}^{\prime}\right)$, for which there is no equivalent $\rho^{\prime}$.

We can now start to formalize what it means for an observation structure to contain enough queries.

Definition 6.8 Let $\langle O b s, \mathcal{N}\rangle$ be an observation structure, let $u_{g} \in \mathcal{N}$, and let $\left[\gamma^{\prime}\right]_{K}$ be an initial region such that $\left[\gamma^{\prime}\right]_{K} \cap s p\left(u_{g}\right) \neq \emptyset$. A clocked word $u_{c}(a, \gamma)$ in $u_{g}$ is a region-representative of $\left[\gamma^{\prime}\right]_{K}$ in $u_{g}$ if $\gamma\left[x_{a} \rightarrow 0\right] \sim_{K} \gamma^{\prime}$ and for every $u_{c}^{\prime} z_{c}^{\prime} \in \operatorname{Dom}($ Obs $)$ with $u_{c}^{\prime} \vDash u_{g}$ and $u_{c}(a, \gamma) \simeq_{K} u_{c}^{\prime}$, there is a $u_{c}(a, \gamma) z_{c} \in \operatorname{Dom}($ Obs $)$ such that $z_{c} \approx_{K} z_{c}^{\prime}$.

Intuitively, a region-representative $u_{c}(a, \gamma)$ of $\left[\gamma^{\prime}\right]_{K}$ in $u_{g}$ has the property that all queries which pass through $u_{g}$, hitting the same initial region as $u_{c}(a, \gamma)$, are also performed with $u_{c}(a, \gamma)$ as prefix. We use the convention that if $u_{g}$ is the root of the tree then $\lambda$ is a region-representative of the (only) initial region $[\gamma]_{K}$ 
in $u_{g}$, which satisfies $\gamma\left(x_{a}\right)=0$ for all $a \in \Sigma$. Note that we do not require that $u_{c}(a, \gamma) \in \operatorname{Dom}(\mathrm{Obs})$.

The following definition formalizes the two properties which together say that an observation structure contains enough queries, stated at the beginning of this subsection.

Definition 6.9 An observation structure $\langle O b s, \mathcal{N}\rangle$ is region-consistent if

- for every node $u_{g} \in \mathcal{N}$ and initial region $\left[\gamma^{\prime}\right]_{K}$ such that $\left[\gamma^{\prime}\right]_{K} \cap \operatorname{sp}\left(u_{g}\right) \neq \emptyset$ there is a region-representative $u_{c}$ of $\left[\gamma^{\prime}\right]_{K}$ in $u_{g}$, and

- for any two nodes $v_{g}$ and $u_{g}$ with $\operatorname{sp}\left(v_{g}\right) \approx_{K} \operatorname{sp}\left(u_{g}\right)$ and height $\left(v_{g}, \mathcal{N}\right) \leq$ height $\left(u_{g}, \mathcal{N}\right)$ we have

- for every initial region $\left[\gamma^{\prime}\right]_{K}$ with $\left[\gamma^{\prime}\right]_{K} \cap s p\left(u_{g}\right) \neq \emptyset$, the regionrepresentative $v_{c}$ of $[\gamma]_{K}$ in $v_{g}$ and the region-representative $u_{c}$ of $[\gamma]_{K}$ in $u_{g}$ have the property that for each $z_{c}$ such that $v_{c} z_{c} \in \operatorname{Dom}(\mathrm{Obs})$ there is a $y_{c}$ with $y_{c} \approx_{K} z_{c}$ such that $u_{c} y_{c} \in \operatorname{Dom}(\mathrm{Obs})$.

Definition 6.10 Let $u_{g} z_{g}$ and $v_{g} z_{g}^{\prime}$ be two guarded words. We say that the suffix $z_{g}$ of $u_{g}$ overlaps with the suffix $z_{g}^{\prime}$ of $v_{g}$ if there are $u_{c} z_{c}$ and $v_{c} z_{c}^{\prime}$ such that $u_{c} z_{c} \vDash u_{g} z_{g}, v_{c} z_{c}^{\prime} \vDash u_{g} z_{g}^{\prime}$ and $z_{c} \approx_{K} z_{c}^{\prime}$.

We introduce a property of a timed decision tree which we call suffixcompleteness, the aim of which is to reduce the number of equivalence queries. It checks, for two nodes that do not agree on overlapping suffixes, that there is indeed some observation that separates them to make sure that the nodes cannot be merged.

Definition 6.11 An observation structure $\langle O b s, \mathcal{N}\rangle$ is suffix-complete if for every $v_{g}, u_{g} \in \mathcal{N}$ with height $\left(v_{g}, \mathcal{N}\right) \leq \operatorname{height}\left(u_{g}, \mathcal{N}\right)$ we have

- untimesuff $_{\mathcal{N}}\left(v_{g}\right) \subseteq$ untimesuff $_{\mathcal{N}}\left(u_{g}\right)$ and

- whenever the suffix $z_{g}$ of $u_{g}$ overlaps with the suffix $z_{g}^{\prime}$ of $v_{g}$, and Obs $\left(v_{g} z_{g}\right) \neq$ Obs $\left(u_{g} z_{g}^{\prime}\right)$, then there are clocked words $v_{c} z_{c}, u_{c} z_{c}^{\prime} \in \operatorname{Dom}($ Obs $)$ with $v_{c} z_{c} \vDash$ $v_{g} z_{g}$ and $u_{c} z_{c}^{\prime} \vDash u_{g} z_{g}^{\prime}$ such that $z_{c} \approx_{K} z_{c}^{\prime}$.

We can now define the second relation of Section 6.1 between nodes, denoted $\preceq_{u n}$, which implies that two nodes can be merged if they have region-equivalent postconditions and agree on suffixes that occur in performed queries.

Definition 6.12 Let $\langle\mathrm{Obs}, \mathcal{N}\rangle$ be a well-formed, consistent, and region-consistent observation structure. Define the relation $\preceq_{u n}$ between nodes by $v_{g} \preceq_{u n} u_{g}$ if

- $s p\left(v_{g}\right) \approx_{K} s p\left(u_{g}\right)$,

- untimesuff $f_{\mathcal{N}}\left(v_{g}\right) \subseteq$ untimesuff $_{\mathcal{N}}\left(u_{g}\right)$, and 
- for any descendants $v_{g} z_{g}$ of $v_{g}$ and $u_{g} z_{g}^{\prime}$ of $u_{g}$ such that the suffix $z_{g}$ of $v_{g}$ overlaps with the suffix $z_{g}^{\prime}$ of $u_{g}$, we have Obs $\left(v_{g} z_{g}\right)=\operatorname{Obs}\left(u_{g} z_{g}^{\prime}\right)$.

Intuitively, $v_{g} \preceq_{u n} u_{g}$ means that all observations represented by the subtree rooted at $v_{g}$ are also represented by the subtree rooted at $u_{g}$. It follows from Definition 6.12 that $\preceq_{u n}$ is height-monotone, transitive, and hence a preorder. Note that $\preceq_{u n}$ differs from $\leq_{u n}$ in that it compares only the parts of subtrees that satisfy the postcondition of the respective nodes: in this way inclusion between subtrees is checked only for the parts in which observations are possible. The following lemma states that comparable nodes must in fact agree on suffixes of performed queries.

Lemma 6.13 Let $\langle O b s, \mathcal{N}\rangle$ be a well-formed, consistent, and region-consistent observation structure. If $v_{g} \preceq_{u n} u_{g}$ then whenever $v_{c} z_{c} \in \operatorname{Dom}(\mathrm{Obs})$ for some $v_{c} \models v_{g}$, there is a $y_{c}$ with $y_{c} \approx_{K} z_{c}$ such that $u_{c} y_{c} \in \operatorname{Dom}($ Obs $)$ for some $u_{c}=u_{g}$ and $\operatorname{Obs}\left(u_{c} y_{c}\right)=\operatorname{Obs}\left(v_{c} z_{c}\right)$.

Proof. The existence of $y_{c}$ follows by region consistency, and $\operatorname{Obs}\left(u_{c} y_{c}\right)=$ $\operatorname{Obs}\left(v_{c} z_{c}\right)$ follows from $v_{g} \preceq_{u n} u_{g}$.

Using Lemma 6.13 , we can now obtain a bound on the size of a $\preceq_{u n}$-unique set of nodes in a timed decision tree, by adapting the corresponding argument for the $L^{*}$-algorithm. This bound allows to prove that if $U$ is a complete, $\preceq_{u n}$-unique, and $\preceq_{u n}$-closed prefix-closed subset of $\mathcal{N}$, then there is a bound on size of a $U_{\leq_{u n}}$-merging. A disadvantage of using the relation $\preceq_{u n}$ when constructing automata, however, is that nodes with postconditions which are not region-equivalent will not be merged. In contrast, the relation $\leq_{u n}$ allows to merge nodes with postconditions which are not region-equivalent, often resulting in significantly smaller automata.

In order to generate automata that are always of bounded size and often small, we therefore present an operation on timed decision trees, called copying. The purpose of this operation is to restructure the timed decision tree $\mathcal{N}$ of an observation structure $\langle O b s, \mathcal{N}\rangle$ so that there exists a $\leq_{u n}$-closed and $\leq_{u n}$-unique prefix of $\mathcal{N}$ which is also $\preceq_{u n}$-unique. This will allow us to prove the same upper bound on the size of $U_{\leq_{u n}}$-mergings as on the size of $U_{\preceq_{u n}}$-mergings.

We define two nodes to be incompatible if they are separated by some suffix.

Definition 6.14 Let $\langle O b s, \mathcal{N}\rangle$ be an observation structure. Two guarded words, $u_{g}$ and $v_{g}$, in $\mathcal{N}$ are incompatible, denoted $u_{g} \star_{K} v_{g}$, if Dom(Obs) contains clocked words $u_{c} z_{c}$ and $v_{c} z_{c}^{\prime}$ with $u_{c} \vDash u_{g}$ and $v_{c} \vDash v_{g}$, such that $z_{c} \approx_{K} z_{c}^{\prime}$ and $\operatorname{Obs}\left(u_{c} z_{c}\right) \neq \operatorname{Obs}\left(v_{c} z_{c}^{\prime}\right)$.

We write $u_{g} \asymp_{K} v_{g}$ to denote that $u_{g}$ and $v_{g}$ are not incompatible (i.e., compatible). 


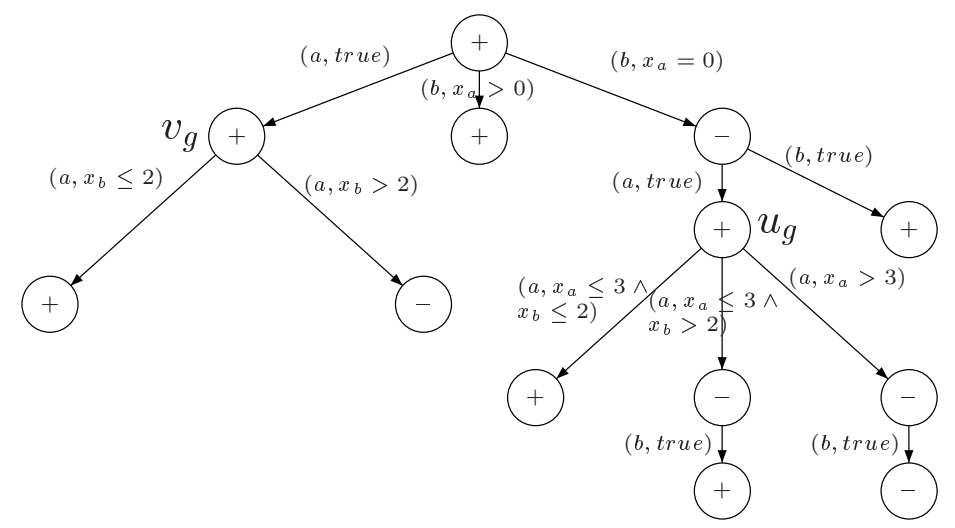

(a) $\mathcal{N}$

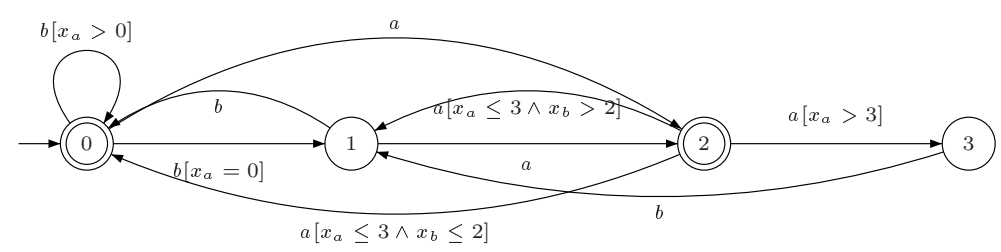

(b) $\mathcal{N}_{\preceq u n}$

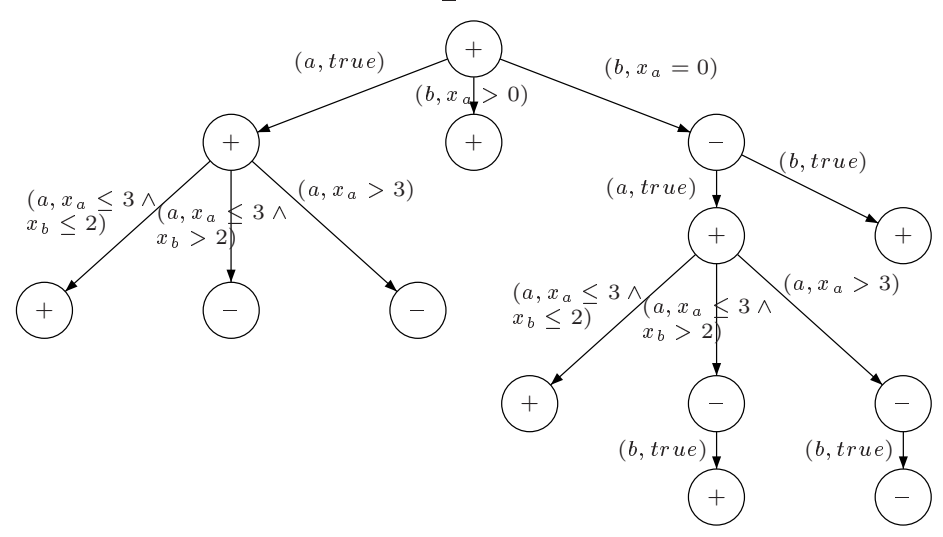

(c) $\operatorname{unfold}_{\mathcal{N}}\left(\mathcal{N}_{\preceq \text { un }}\right)$

Figure 6: Copying subtrees 


\subsection{The Copying Operation}

In this section we describe the copying operation which is performed before the construction in Theorem 6.5, in order to guarantee a bounded size on the resulting automaton. The copying operation restructures the timed decision tree $\mathcal{N}$ of an observation structure $\langle O b s, \mathcal{N}\rangle$ so that there exists a $\leq_{u n}$-closed and $\leq_{u n}$-unique prefix of $\mathcal{N}$ which is also $\preceq_{u n}$-unique. The construction assumes that we have found a $\preceq_{u n}$-closed and $\preceq_{u n}$-unique prefix $V$ of $\mathcal{N}$. This means that for any node $v_{g}$ in $\operatorname{succ}(V)$, there is a $u_{g}$ in $V$ with $v_{g} \preceq_{u n} u_{g}$. However, it is not guaranteed that there is a $u_{g}$ in $V$ with $v_{g} \leq_{u n} u_{g}$. The goal of the copying operation is to restructure the subtree rooted at $v_{g}$ by "copying" so that $v_{g} \leq_{u n} u_{g}$ for some $u_{g} \in V$. As a result, $V$ will become $\leq_{u n}$-closed, and we can thereafter search for a $\leq_{u n}$-closed and $\leq_{u n}$-unique prefix-closed subset $U$ of $V$, which by construction will also be $\preceq u n$-unique. This will allow us to prove the same upper bound on the size of $U_{\leq_{u n}}$-mergings as on the size of $U \preceq_{u n}$-mergings.

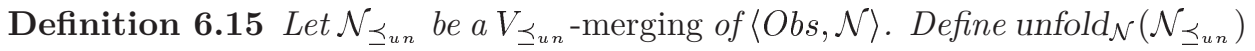
to be the timed decision tree such that $w_{g} \in \operatorname{unfold}_{\mathcal{N}}\left(\mathcal{N}_{\preceq_{u n}}\right)$ iff $\lambda \stackrel{w_{g}}{\Rightarrow} l$ in $\mathcal{N}_{\preceq_{u n}}$ for some location $l$ in $\mathcal{N}_{\preceq_{u n}}$, and there is some $w_{g}^{\prime} \in \mathcal{N}$ with untime $\left(w_{g}\right)^{-}=$ untime $\left(w_{g}^{\prime}\right)$.

Intuitively, if we have found a $\preceq_{u n}$-closed and $\preceq_{u n}$-unique prefix-closed set of nodes $V$, then the copying operation folds $\mathcal{N}$ by merging nodes which are equivalent wrp. to $\preceq_{\text {un }}$, whereafter $\mathcal{N}$ is again unfolded. Note that $V$ may contain leaves.

Example 6.16 To illustrate the copying operation, consider the timed decision tree shown in Figure 6(a). It has two nodes $v_{g}=(a, t r u e)$ and $u_{g}=\left(b, x_{a}=\right.$ $0)(a$, true $)$ such that $s p\left(v_{g}\right)$ and $s p\left(u_{g}\right)$ are both $x_{a} \leq x_{b}$. Since Obs $\left(v_{g}\left(a, x_{b} \leq\right.\right.$ $2)) \neq \operatorname{Obs}\left(u_{g}\left(a, x_{a}>3\right)\right)$ and suffixes $\left(a, x_{b} \leq 2\right)$ and $\left(a, x_{a}>3\right)$ are suffixoverlapped, it follows that $v_{g} \mathbb{Z}_{u n} u_{g}$. On the other hand, if we restrict the subtrees rooted at $u_{g}$ and $v_{g}$ to suffixes that can actually be part of clocked words, then they do not disagree, i.e., $v_{g} \preceq_{u n} u_{g}$. In order to make $v_{g}$ unifiable with $u_{g}$, the copying procedure copies the subtree rooted at $u_{g}$ to the subtree rooted at $v_{g}$, obtaining the timed decision tree shown in Figure 6(c).

The following lemma implies that after the copying operation preserves consistency of a well-formed tree, implying that after copying, we can use Theorem 6.5 to construct a TDERA that agrees with $O b s$.

Lemma 6.17 Let $\langle\mathrm{Obs}, \mathcal{N}\rangle$ be a well-formed and consistent observation struc-

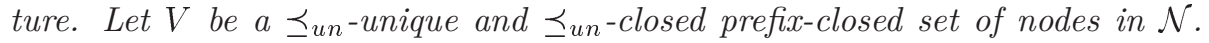
Construct $\mathcal{N}^{\prime}=\operatorname{unfold}_{\mathcal{N}}\left(\mathcal{N}_{\preceq_{u n}}\right)$ as in Definition 6.15. Then $\left\langle O b s, \mathcal{N}^{\prime}\right\rangle$ is a consistent observation structure. 
Proof. It follows directly that nodes in $V \cup \operatorname{succ}(V)$ are consistent. We prove, using induction over the length of the guarded word $z_{g}$, that whenever $v_{g} \in$ $V \cup \operatorname{succ}(V)$, then for any descendants $v_{g} y_{g}, v_{g} y_{g}^{\prime} \in \mathcal{N}$ and $v_{g} z_{g} \in \mathcal{N}^{\prime}$ such that the suffix $z_{g}$ of $v_{g}$ overlaps with the suffix $y_{g}$ of $v_{g}$ and such that the suffix $z_{g}$ of $v_{g}$ overlaps with the suffix $y_{g}^{\prime}$ of $v_{g}$, we have $\operatorname{Obs}\left(v_{g} y_{g}\right)=\operatorname{Obs}\left(v_{g} y_{g}^{\prime}\right)$, and hence $v_{g} z_{g}$ must be a consistent node.

We first consider the case $v_{g} \in \operatorname{succ}(V)$. Let us use the notation $\left(v_{g}, \mathcal{N}\right)$ to stress that we consider a node $v_{g}$ which belongs to the tree $\mathcal{N}$. The case where $z_{g}=y_{g}=y_{g}^{\prime}=\lambda$ follows trivially. So, assume that $z_{g} \neq \lambda$. If $y_{g}=y_{g}^{\prime}$, we are done. Otherwise, by Definition 6.3 there is $u_{g} \in V$, such that $\left(v_{g}, \mathcal{N}\right) \preceq_{u n}$ $\left(u_{g}, \mathcal{N}\right)$, i.e., $s p\left(v_{g}\right) \approx_{K} s p\left(u_{g}\right)$ and $v_{g}$ is merged with $u_{g}$ when constructing $\mathcal{N}_{\preceq_{u n}}$. Then either

- $u_{g} z_{g} \in V \cup \operatorname{succ}(V)$, in which case the claim follows by observing that $\left(v_{g}, \mathcal{N}\right) \preceq_{u n}\left(u_{g}, \mathcal{N}\right)$ implies that $\operatorname{Obs}\left(v_{g} y_{g}\right)=\operatorname{Obs}\left(u_{g} z_{g}\right)=\operatorname{Obs}\left(v_{g} y_{g}^{\prime}\right)$, since from $s p\left(v_{g}\right) \approx_{K} s p\left(u_{g}\right)$ we have that the suffix $z_{g}$ of $u_{g}$ overlaps with the suffix $y_{g}$ of $v_{g}$ and the suffix $z_{g}$ of $u_{g}$ overlaps with the suffix $y_{g}^{\prime}$ of $v_{g}$, or

- there is a prefix $u_{g} v_{g}^{\prime} \in \operatorname{succ}(V)$ of $u_{g} z_{g}$. Since the suffix $y_{g}$ of $v_{g}$ overlaps with suffix $z_{g}$ of $v_{g}$ and $s p\left(v_{g}\right) \approx_{K} s p\left(u_{g}\right)$ we infer that the suffix $y_{g}$ of $u_{g}$ overlaps with the suffix $z_{g}$ of $u_{g}$. Then there is a node $u_{g} v_{g}^{\prime} x_{g} \in \mathcal{N}$ such that the suffix $v_{g}^{\prime} x_{g}$ of $u_{g}$ overlaps with the suffix $y_{g}$ of $v_{g}$ and the suffix $v_{g}^{\prime} x_{g}$ of $u_{g}$ overlaps with the suffix $z_{g}$ of $u_{g}$, from which we use $\left(v_{g}, \mathcal{N}\right) \preceq_{u n}\left(u_{g}, \mathcal{N}\right)$ to infer $\operatorname{Obs}\left(u_{g} v_{g}^{\prime} x_{g}\right)=\operatorname{Obs}\left(v_{g} y_{g}\right)$. In an analogous manner, we infer that there is a node $u_{g} v_{g}^{\prime} x_{g}^{\prime} \in \mathcal{N}$ such that the suffix $v_{g}^{\prime} x_{g}^{\prime}$ of $u_{g}$ overlaps with the suffix $y_{g}^{\prime}$ of $v_{g}$ and the suffix $v_{g}^{\prime} x_{g}^{\prime}$ of $u_{g}$ overlaps with the suffix $z_{g}$ of $u_{g}$, and $\operatorname{Obs}\left(u_{g} v_{g}^{\prime} x_{g}^{\prime}\right)=\operatorname{Obs}\left(v_{g} y_{g}^{\prime}\right)$. Let $u_{g} z_{g}=u_{g} v_{g}^{\prime} z_{g}^{\prime}$. Since the suffix $v_{g}^{\prime} x_{g}$ of $u_{g}$ overlaps with the suffix $z_{g}$ of $u_{g}$ we infer that the suffix $x_{g}$ of $u_{g} v_{g}^{\prime}$ overlaps with the suffix $z_{g}^{\prime}$ of $u_{g} v_{g}^{\prime}$. Moreover, since the suffix $v_{g}^{\prime} x_{g}^{\prime}$ of $u_{g}$ overlaps with the suffix $z_{g}$ of $u_{g}$ we infer that the suffix $x_{g}^{\prime}$ of $u_{g} v_{g}^{\prime}$ overlaps with the suffix $z_{g}^{\prime}$ of $u_{g} v_{g}^{\prime}$. By the inductive hypothesis, since $z_{g}^{\prime}$ is shorter than $z_{g}$, we infer that $\operatorname{Obs}\left(u_{g} v_{g}^{\prime} x_{g}\right)=\operatorname{Obs}\left(u_{g} v_{g}^{\prime} x_{g}^{\prime}\right)$. Then $O b s\left(v_{g} y_{g}\right)=\operatorname{Obs}\left(v_{g} y_{g}^{\prime}\right)$.

It is straightforward to use this claim to establish the claim for nodes $v_{g} \in V$.

Lemma 6.18 Let $\langle O b s, \mathcal{N}\rangle$ be a well-formed, consistent, and region-consistent

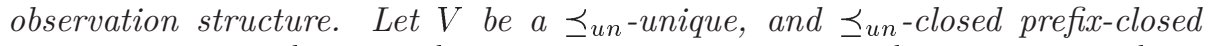
set of nodes in $\mathcal{N}$. Let $\mathcal{N}_{\preceq_{u n}}$ be a $V_{\preceq_{u n}}$-merging of $\mathcal{N}$. Construct $\mathcal{N}^{\prime}=$ unfold $_{\mathcal{N}}\left(\mathcal{N}_{\preceq_{u n}}\right)$ as in Definition 6.15. Then $V$ is $\leq_{u n}$-closed in $\mathcal{N}^{\prime}$.

Proof. Let $\mathcal{N}_{\preceq_{u n}}=\left\langle U,\{\lambda\}, L^{f}, E\right\rangle$. We prove that if $u_{g} \in V, v_{g}=u_{g}^{\prime}(a, g) \in$ $\operatorname{succ}(V),\left(v_{g}, \mathcal{N}\right) \preceq_{u n}\left(u_{g}, \mathcal{N}\right)$, and there is $\left(u_{g}^{\prime}, u_{g}, a, g\right) \in E$ then $\left(v_{g}, \mathcal{N}^{\prime}\right) \leq_{u n}$ $\left(u_{g}, \mathcal{N}^{\prime}\right)$. Namely, then for every node $v_{g} z_{g} \in \mathcal{N}^{\prime}$ it follows, from Lemma 6.13, that there is a node $u_{g} z_{g} \in \mathcal{N}^{\prime}$ such that $\operatorname{Obs}\left(v_{g} z_{g}\right)=\operatorname{Obs}\left(u_{g} z_{g}\right)$, from which we infer $\left(v_{g}, \mathcal{N}^{\prime}\right) \leq{ }_{u n}\left(u_{g}, \mathcal{N}^{\prime}\right)$. 
We remark that if $V$ is complete in $\mathcal{N}$, then it is also complete in $\mathcal{N}^{\prime}$. Copying can destroy well-formedness of the tree, but well-formedness is not required when constructing a TDERA in Definition 6.3.

From Lemma 6.18 it follows that there is a $\leq_{u n}$-closed and $\leq_{u n}$-unique

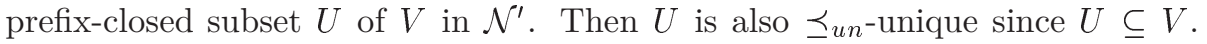
To make $U$ complete, whenever $u_{g} \in U$ and $a \in \Sigma$ are such that there is no $u_{g}(a, g) \in \mathcal{N}^{\prime}$, we add $u_{g}(a$, true $)$ to $\mathcal{N}^{\prime}$ and ask membership query for $u_{c}$ with $u_{c} \vDash u_{g}($ a,true $)$. Furthermore, the extra node $u_{g}(a$, true $)$ satisfies $u_{g}(a$, true $) \leq_{u n} u_{g}^{\prime}$ for some $u_{g}^{\prime} \in U$ implying that the addition preserves $\leq_{u n^{-}}$ closedness of $U$, which is required by Definition 6.3.

\section{The Overall Algorithm}

We can now finally bring all pieces together and present the overall algorithm for inferring a TDERA. The algorithm is represented by the function Learner, described in Algorithm 2.

Lines $2-3$ perform initialization of the observation structure by constructing the initial timed decision tree with the empty word as root and children for each symbol in the alphabet. Lines $5-38$ contain the steps for constructing a well-formed, consistent, region-consistent, and suffix-complete observation structure. Here lines $6-9$ show the steps for achieving well-formedness, lines $10-14$ show the steps for achieving consistency, lines $15-27$ are concerned with achieving region-consistency and lines 28-37 are concerned with achieving suffix-completeness. After having constructed a well-formed, consistent, regionconsistent and suffix-complete observation structure, lines 40 - 48 construct a hypothesis TDERA to prepare an equivalence query. Here line 40 finds a suitable prefix $V$, whereafter the copying operation is performed in lines $41-42$. Lines 43-47 finds a suitable prefix $U$ of $V$, and after fixing possible problems with $U$ being not complete, the hypothesis $\mathcal{H}$ is formed in line 48. The equivalence query is posed in line 50. If it is successful, the algorithm terminates, otherwise the counterexample is inserted into the observation structure and the algorithm is restarted from line 5 .

\subsection{Termination proof and complexity results}

In this section, we prove that our inference algorithm terminates after a bounded number of membership and equivalence queries. As a first step, we prove an upper bound on the number of locations in any constructed automaton.

Lemma 7.1 Let $\langle O b s, \mathcal{N}\rangle$ be a well-formed, consistent, and region-consistent observation structure. Let $V$ be $a \preceq$ un-unique set of nodes in $\mathcal{N}$. Then $V$ has at most $|L+1|^{R_{K}}$ nodes, where $|L|$ is the number of locations of $\mathcal{A}$ and $R_{K}$ is the number of regions.

Proof. Let the TDERA $\mathcal{A}$ be $\left\langle L,\left\{l^{0}\right\}, L^{f}, E\right\rangle$. Assign to each node $u_{g} \in V$ a mapping $\theta\left[u_{g}\right]$ from initial regions that intersect $s p\left(u_{g}\right)$ to $L \cup\{\perp\}$, as follows. 


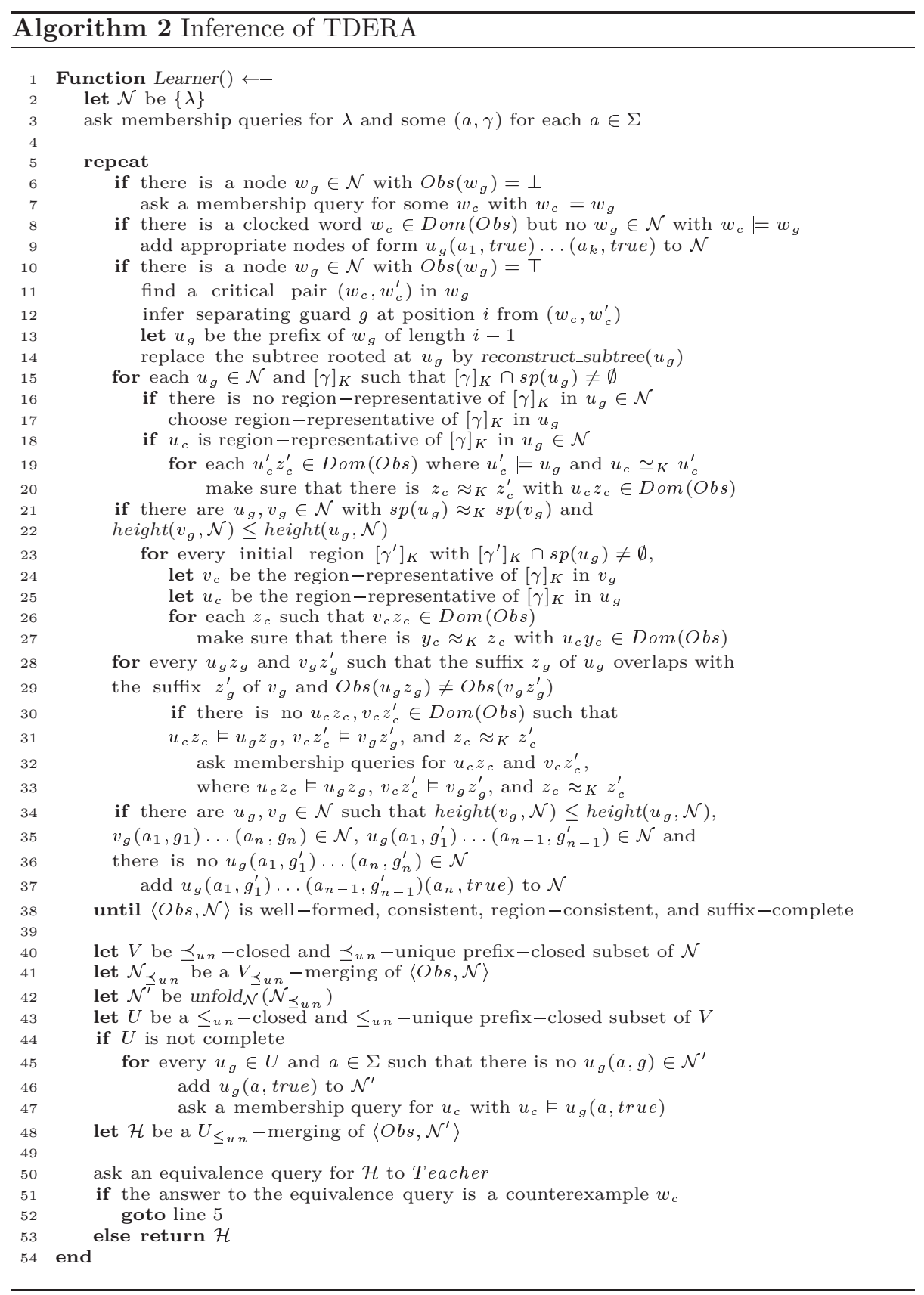


For an initial region $[\gamma]_{K}$ such that $[\gamma]_{K} \cap s p\left(u_{g}\right) \neq \emptyset$, let $\theta\left[u_{g}\right]\left([\gamma]_{K}\right)=l$ if there is a region-representative $u_{c}$ of $[\gamma]_{K}$ in $u_{g}$, such that $u_{c}$ leads to the location $l \in L$ in $\mathcal{A}$, i.e., such that $l^{0} \stackrel{w_{g}}{\Rightarrow} l$ in $\mathcal{A}$ for some $w_{g}$ with $u_{c} \models w_{g}$. Let $\theta\left[u_{g}\right]\left([\gamma]_{K}\right)=\perp$ if $[\gamma]_{K} \cap s p\left(u_{g}\right)=\emptyset$. Note that region-consistency does not require that there is some $u_{c} y_{c} \in \operatorname{Dom}(\mathrm{Obs})$, but still every $u_{c}$ leads to some location in $\mathcal{A}$. We next observe that if $\theta\left[u_{g}\right]\left([\gamma]_{K}\right)=\theta\left[u_{g}^{\prime}\right]\left([\gamma]_{K}\right) \neq \perp$ for an initial region $[\gamma]_{K}$, i.e., both $u_{g}$ and $u_{g}^{\prime}$ have region-representatives of $[\gamma]_{K}$ which lead to the same location $l$, then whenever $u_{c} z_{c}, u_{c}^{\prime} z_{c}^{\prime} \in \operatorname{Dom}(\mathrm{Obs})$ such that $u_{c} \vDash u_{g}, u_{c}^{\prime} \vDash u_{g}^{\prime}$, and $z_{c} \approx_{K} z_{c}^{\prime}$, then $\operatorname{Obs}\left(u_{c} z_{c}\right)=\operatorname{Obs}\left(u_{c}^{\prime} z_{c}^{\prime}\right)$. It follows that if $\theta\left[u_{g}\right]$ and $\theta\left[u_{g}^{\prime}\right]$ agree on all initial regions, then $\operatorname{sp}\left(u_{g}\right) \approx_{K} \operatorname{sp}\left(u_{g}^{\prime}\right)$ and $u_{g} \asymp_{K} u_{g}^{\prime}$. Since $\langle O b s, \mathcal{N}\rangle$ is region-consistent, then $u_{g} \preceq_{u n} u_{g}^{\prime}$ or $u_{g}^{\prime} \preceq_{u n} u_{g}$. Since $V$ is $\preceq_{u n}$-unique, the size of $V$ is at most $|L+1|^{R_{K}}$ nodes.

Theorem 7.2 Algorithm 2 terminates.

Proof. To prove termination of Algorithm 2 we should prove that the repeatuntil loop in lines 5 - 38 terminates, and that there is a bound on the number of unsuccessful equivalence queries posed in line 50. Termination of the repeatuntil loop follows by observing that no operation increases the depth of the tree, and that the number of possible timed decision trees of a given depth is bounded.

From Theorem 6.5 follows that every hypothesised automaton $\mathcal{H}$ which is constructed agrees with Obs. Thus every counterexample which is returned in an unsuccessful equivalence query is new and every constructed hypothesis $\mathcal{H}$ is different from any previously constructed hypothesis. From Lemma 7.1 follows that the size of $\mathcal{H}$ is bounded. Since the number of different TDERAs of bounded size is bounded, then the outer loop in function Learner terminates.

A more precise (but still very large) bound on the number of queries is established in the following theorem.

Theorem 7.3 The algorithm for learning TDERA performs $O\left(\left(\frac{(|\Sigma|+2 K+1) e}{\Sigma}\right)^{|\Sigma|(l+1)}\right)$ queries, where $l$ is the length of the longest counterexample and $e$ is the base of natural logarithm.

Proof. An equivalence query introduces at least one new node into the tree. Since the postcondition defines at least an ordering on clocks, then a node can have at most $\left(\begin{array}{c}|\Sigma|+2 K+1 \\ |\Sigma|\end{array}\right)$ children. This is the number of non-increasing sequences of $|\Sigma|$ elements, where each element has values among 0 to $2 K+1$. The height of the tree is at most $l+1$. From [Od195] we know that $\left(\begin{array}{c}|\Sigma|+2 K+1 \\ |\Sigma|\end{array}\right) \leq$ $\left(\frac{(|\Sigma|+2 K+1) e}{\Sigma}\right)^{|\Sigma|}$. It follows that the number of nodes in the final tree, and hence the number of equivalence queries is $O\left(\left(\frac{(|\Sigma|+2 K+1) e}{\Sigma}\right)^{|\Sigma|(l+1)}\right)$. A membership query is performed at most once for every simple guarded word of length at most $l+1$. Then the number of membership queries is $O\left(\left(\frac{(|\Sigma|+2 K+1) e}{\Sigma}\right)^{|\Sigma|(l+1)}\right)$. 


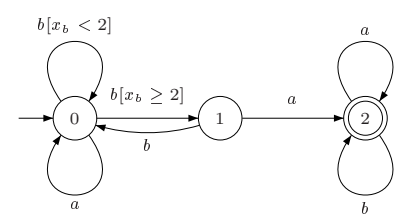

(a) $\mathcal{A}$

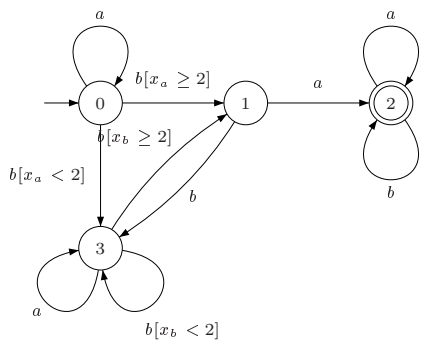

(b) $\mathcal{A}^{\prime}$

Figure 7: Automata $\mathcal{A}$ and $\mathcal{A}^{\prime}$

A bound on the parameter $l$ in Theorem 7.3 can be obtained by assuming that returned counterexamples are as short as possible.

Theorem 7.4 Let $|L|$ be the number of locations in $\mathcal{A}$. Assume that unsuccessful equivalence queries return a shortest (in number of symbols) counterexample. Then the length of any counterexample is less than $2|L| R_{K}$ where $R_{K}$ is the number of regions.

Proof. It was shown in [GJL05] that $\mathcal{A}$ can be transformed into an equivalent simple TDERA $\mathcal{A}^{\prime}$ with at most $|L| R_{K}$ states. Then inference algorithm for deterministic finite automata can be applied to learn $\mathcal{A}^{\prime}$. It follows from [TB73] that the length of counterexamples is less than $2|L| R_{K}$.

For comparison, in [GJL05] is presented an algorithm that infers the region graph of some automaton equivalent to $\mathcal{A}$. The number of equivalence queries is at most $|L| R_{K}$ and the number of membership queries is $O\left(|\Sigma|^{2}|L|^{2} R_{K}^{2} l K\right)$, where $R_{K}$ is the number of regions, $l$ is the length of longest counterexample and $L$ is the number of locations in $\mathcal{A}$.

\section{Example}

Suppose the automaton to be learned is the event-recording automaton $\mathcal{A}$ in Figure $7(\mathrm{a})$. We assume that we know that $K=2$. We start by asking membership queries for $\lambda,(a, 0)$ and $(b, 0)$. Then we construct the timed decision tree $\mathcal{N}_{1}$ shown in Figure 8(a). Then $V=\{\lambda\}$ and $\mathcal{N}_{1}^{\prime}=\mathcal{N}_{1}$, i.e. $\mathcal{N}_{1}$ is not changed after copying. Then $U=\{\lambda\}$ is $\leq_{u n^{-}}$-closed, $\leq_{u n^{-}}$-unique, complete and $\preceq_{u n^{-}}$ unique prefix-closed subset of $V$ in $\mathcal{N}_{1}^{\prime}$. The Learner constructs hypothesized automaton $\mathcal{H}_{1}$ that is $U_{\leq_{u n}}$-merging of $\left\langle O b s, \mathcal{N}_{1}^{\prime}\right\rangle$, shown in Figure 8(b).

Assume that the counterexample $(b, 2)(a, 2.4)$ is returned. It is accepted by $\mathcal{A}$ but rejected by $\mathcal{H}_{1}$. Then we construct the tree $\mathcal{N}_{2}$, shown in Figure 9 (a).

The observation structure $\left\langle O b s, \mathcal{N}_{2}\right\rangle$ is not suffix-complete, since the suffix $(a$, true $)$ of $\lambda$ overlaps with the suffix $(a$, true $)$ of $(b$, true $), \operatorname{Obs}((a$, true $)) \neq$ 
$\operatorname{Obs}((b$, true $)(a$, true $))$, and there are no $u_{c}, u_{c}^{\prime} z_{c} \in \operatorname{Dom}($ Obs $)$ such that $u_{c}$ F (a,true), $u_{c}^{\prime} z_{c} \vDash(b$, true $)(a$, true $)$ and $u_{c} \approx_{K} z_{c}$. We ask membership query for $(b, 0)(a, 0)$. Since $(b, 0)(a, 0)$ is rejected by $\mathcal{A}$, then the node $(b$, true $)(a$, true $)$ becomes inconsistent. We perform binary search and ask membership queries for $(b, 1)(a, 1.2),(b, 1.5)(a, 1.8)$ and $(b, 1.75)(a, 2.1)$. Then we have the critical pair

$$
\begin{aligned}
& \left(b,\left(\begin{array}{c}
x_{a} \rightarrow 2 \\
x_{b} \rightarrow 2
\end{array}\right)\right)\left(a,\left(\begin{array}{c}
x_{a} \rightarrow 2.4 \\
x_{b} \rightarrow 0.4
\end{array}\right)\right) \text { and } \\
& \left(b,\left(\begin{array}{c}
x_{a} \rightarrow 1.75 \\
x_{b} \rightarrow 1.75
\end{array}\right)\right)\left(a,\left(\begin{array}{c}
x_{a} \rightarrow 2.1 \\
x_{b} \rightarrow 0.35
\end{array}\right)\right)
\end{aligned}
$$

from which we infer separating guards $x_{a} \geq 2$ and $x_{b} \geq 2$ at position 1 . At this point we (arbitrarily) choose inequality $x_{a} \geq 2$ and construct timed decision tree $\mathcal{N}_{3}$, shown in Figure 9(b), by splitting node $(b$, true $)$. Then $\left\langle O b s, \mathcal{N}_{3}\right\rangle$ is consistent. Since the suffix (a,true) of $\lambda$ overlaps with the suffix $(a$, true) of $\left(b, x_{a} \geq 2\right), \operatorname{Obs}((a$, true $)) \neq \operatorname{Obs}\left(\left(b, x_{a} \geq 2\right)(a\right.$, true $\left.)\right)$, and there are no $u_{c}$ and $u_{c}^{\prime} z_{c}$ such that $u_{c} \vDash(a$, true $), u_{c}^{\prime} z_{c} \vDash\left(b, x_{a} \geq 2\right)(a$, true $)$ and $u_{c} \approx_{K} z_{c}$ then $\left\langle\mathrm{Obs}, \mathcal{N}_{3}\right\rangle$ is not suffix-complete. Then we ask membership queries for $(a, 2.4)$ and $(b, 2)(a, 4.4)$.

Let $(b, 1.5)$ be a region-representative of $[\gamma]_{K}$ in $\left(b, x_{a}<2\right)$, where $\gamma\left(x_{a}\right)=$ $\gamma\left(x_{b}\right)=1.5$. Then $\left\langle\mathrm{Obs}, \mathcal{N}_{3}\right\rangle$ is suffix-complete, but not region-consistent, since we have observations

$$
\begin{aligned}
& u_{c} z_{c}=\left(b,\left(\begin{array}{c}
x_{a} \rightarrow 1.5 \\
x_{b} \rightarrow 1.5
\end{array}\right)\right)\left(a,\left(\begin{array}{c}
x_{a} \rightarrow 1.8 \\
x_{b} \rightarrow 0.3
\end{array}\right)\right) \text { and } \\
& u_{c}^{\prime} z_{c}^{\prime}=\left(b,\left(\begin{array}{c}
x_{a} \rightarrow 1.75 \\
x_{b} \rightarrow 1.75
\end{array}\right)\right)\left(a,\left(\begin{array}{c}
x_{a} \rightarrow 2.1 \\
x_{b} \rightarrow 0.35
\end{array}\right)\right)
\end{aligned}
$$

where $u_{c} \neq \lambda, z_{c} \neq \lambda, u_{c}^{\prime} \neq \lambda, z_{c}^{\prime} \neq \lambda$ and $u_{c} \simeq_{K} u_{c}^{\prime}$, but $z_{c} \not_{K} z_{c}^{\prime}$. Let

$$
u_{c} z_{c}^{\prime \prime}=\left(b,\left(\begin{array}{c}
x_{a} \rightarrow 1.5 \\
x_{b} \rightarrow 1.5
\end{array}\right)\right)\left(a,\left(\begin{array}{c}
x_{a} \rightarrow 2.1 \\
x_{b} \rightarrow 0.6
\end{array}\right)\right)
$$

Since $u_{c} z_{c}^{\prime \prime} \approx_{K} u_{c}^{\prime} z_{c}^{\prime}$ then $u_{c} z_{c}^{\prime \prime}$ is accepted iff $u_{c}^{\prime} z_{c}^{\prime}$ is accepted. Then we do not need to ask membership query for $u_{c} z_{c}^{\prime \prime}$. To make $\left\langle O b s, \mathcal{N}_{3}\right\rangle$ region-consistent we add $u_{c} z_{c}^{\prime \prime}$ to $\operatorname{Dom}(\mathrm{Obs})$.

We have $\left(b, x_{a}<2\right)(a$, true $) \preceq$ un $(a$, true $)$. Then $V=\left\{\lambda,(a\right.$, true $),\left(b, x_{a}<\right.$ $2),\left(b, x_{a} \geq 2\right),\left(b, x_{a} \geq 2\right)(a$, true $\left.)\right\}$ is $\preceq_{u n}$-closed and $\preceq_{u n}$-unique prefix-closed subset of $\mathcal{N}_{3}$. Then $\mathcal{N}_{3}^{\prime}=\mathcal{N}_{3}$, i.e. $\mathcal{N}_{3}$ is not changed after copying. To make $U \leq u_{u n}$-closed, we add $\left(b, x_{a} \geq 2\right)$ and $\left(b, x_{a} \geq 2\right)(a$, true $)$ to $U$. To make

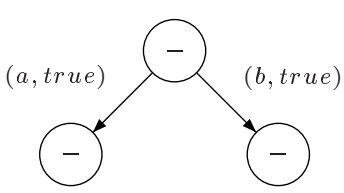

(a) $\mathcal{N}_{1}$

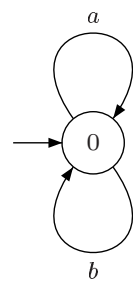

(b) $\mathcal{H}_{1}$

Figure 8: A tree $\mathcal{N}_{1}$ and an automaton $\mathcal{H}_{1}$ 


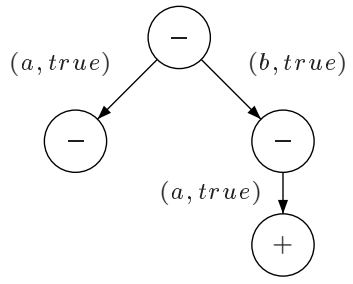

(a) $\mathcal{N}_{2}$

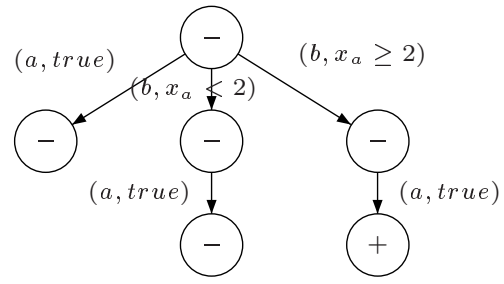

(b) $\mathcal{N}_{3}$

Figure 9: Trees $\mathcal{N}_{2}$ and $\mathcal{N}_{3}$

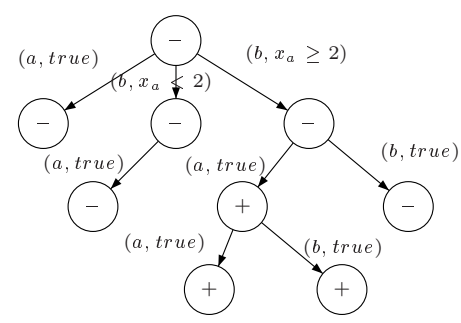

(a) $\mathcal{N}_{3}^{\prime}$

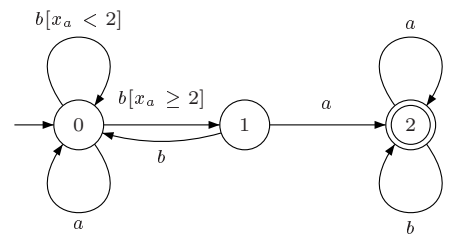

(b) $\mathcal{H}_{2}$

Figure 10: A tree $\mathcal{N}_{3}^{\prime}$ and an automaton $\mathcal{H}_{2}$

$U$ complete we add $\left(b, x_{a} \geq 2\right)(a$, true $)(a$, true $),\left(b, x_{a} \geq 2\right)(a$, true $)(b$, true $)$ and $\left(b, x_{a} \geq 2\right)(b$, true $)$ to $\mathcal{N}_{3}^{\prime}$ and ask membership queries for $(b, 2)(b, 2)$, $(b, 2)(a, 2)(a, 2)$, and $(b, 2)(a, 2)(b, 2)$. The resulting timed decision tree $\mathcal{N}_{3}^{\prime}$ is

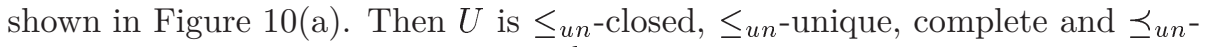
unique prefix-closed subset of $V$ in $\mathcal{N}_{3}^{\prime}$.

The Learner construct hypothesized automaton $\mathcal{H}_{2}$ that is $U_{\leq_{u n}}$-merging of $\left\langle O b s, \mathcal{N}_{3}^{\prime}\right\rangle$, shown in Figure 10(b). The procedure continues and the Learner will construct three more automata before the automaton $\mathcal{A}^{\prime}$, shown in Figure 7 (b), is finally created.

\section{Conclusion}

We have presented a technique for inference of timed systems that can be represented as event-recording automata. We introduced timed decision tree as a data structure for organizing the results of the membership and equivalence queries. The timed decision tree is folded into an event-recording automaton by a merging procedure that is based on a notion of unifiable nodes. This is the first inference algorithm for the full class of event-recording automata which avoids explicit use of the region graph. The algorithm works under assumption 
that a greatest constant $K$ which appears in clock guards is known. To prove termination of the algorithm we introduced region-representatives. The number of region-representatives depends on $K$, and it is not clear how to prove termination of the algorithm if $K$ is unknown.

The drawback of the algorithm that it has high complexity. However, the algorithm can be modified in such a way that it can learn DERAs with one clock by asking only a polynomial number of membership and equivalence queries. It would be interesting to find a class of DERAs with two clocks for which a smallest automaton can be learned by asking polynomial number of membership and equivalence queries. A difference between timed automata with one and two clocks is shown in [LMS04]. Laroussinie et al. present the polynomial algorithm for model checking $T C T L_{\leq, \geq}$over timed automata with one clock and show that model checking $C T L$ over timed automata with two clocks is PSPACEcomplete.

Another interesting problem is to develop algorithm for learning deterministic timed automata. Difference between timed automata and DERAs that we do not know how many clocks a timed automaton has and they can reset at any transition. We can construct a timed decision tree by introducing at every transition a new clock, but then it is not clear how a merging procedure should work for constructing a timed automaton from the timed decision tree, since we need to compare edges which are labeled by guards containing different clocks.

\section{References}

[AD94] R. Alur and D. Dill. A theory of timed automata. Theoretical Computer Science, 126:183-235, 1994.

[AFH99] R. Alur, L. Fix, and T. Henzinger. Event-clock automata: A determinizable class of timed automata. Theoretical Computer Science, 211:253-273, 1999.

[Ang87] Dana Angluin. Learning regular sets from queries and counterexamples. Information and Computation, 75:87-106, 1987.

[BDG97] José L. Balcázar, Josep Díaz, and Ricard Gavaldá. Algorithms for learning finite automata from queries: A unified view. In Advances in Algorithms, Languages, and Complexity, pages 53-72. Kluwer, 1997.

$\left[\mathrm{BDM}^{+} 98\right]$ M. Bozga, C. Daws, O. Maler, A. Olivero, S. Tripakis, and S. Yovine. Kronos: A model-checking tool for real-time systems. In A. J. Hu and M. Y. Vardi, editors, Proc. CAV'98, volume 1427 of LNCS, pages 546-550. Springer-Verlag, 1998.

$\left[\mathrm{BGJ}^{+} 05\right]$ Therese Berg, Olga Grinchtein, Bengt Jonsson, Martin Leucker, Harald Raffelt, and Bernhard Steffen. On the correspondence between conformance testing and regular inference. In Proc. of 8th 
International Conference on Fundamental Approaches to Software Engineering, pages 175-189, 2005.

$\left[\mathrm{BLL}^{+} 96\right]$ J. Bengtsson, K. G. Larsen, F. Larsson, P. Pettersson, and Wang Yi. UPPAAL: a tool suite for the automatic verification of realtime systems. In R. Alur, T. A. Henzinger, and E. D. Sontag, editors, Hybrid Systems III, volume 1066 of LNCS, pages 232-243. Springer-Verlag, 1996.

$\left[\mathrm{CDH}^{+} 00\right] \quad$ J.C. Corbett, M.B. Dwyer, J. Hatcliff, S. Laubach, C.S. Pasareanu, Robby, and H. Zheng. Bandera : Extracting finite-state models from java source code. In Proc. 22nd Int. Conf. on Software Engineering, June 2000.

[CGP99] E.M. Clarke, O. Grumberg, and D. Peled. Model Checking. MIT Press, Dec. 1999.

[Cho78] Tsun S. Chow. Testing software design modeled by finite-state machines. IEEE Trans. Software Eng., 4(3):178-187, 1978.

[Dil89] David L. Dill. Timing assumptions and verification of finite-state concurrent systems. In Automatic Verification Methods for Finite State Systems, pages 197-212, 1989.

[DT98] Conrado Daws and Stavros Tripakis. Model checking of real-time reachability properties using abstractions. In TACAS, pages $313-$ $329,1998$.

[FJJV97] J.-C. Fernandez, C. Jard, T. Jéron, and C. Viho. An experiment in automatic generation of test suites for protocols with verification technology. Science of Computer Programming, 29, 1997.

[GJL04] Olga Grinchtein, Bengt Jonsson, and Martin Leucker. Learning of event-recording automata. In Proceedings of the Joint Conferences FORMATS and FTRTFT, volume 3253 of LNCS, September 2004.

[GJL05] Olga Grinchtein, Bengt Jonsson, and Martin Leucker. Inference of timed transition systems. ENCS, 138(3):87-99, 2005.

[Gol67] E. M. Gold. Language identification in the limit. Information and Control, 10:447-474, 1967.

[GPY02] Alex Groce, Doron Peled, and Mihalis Yannakakis. Adaptive model checking. In Proc. TACAS'02, LNCS, 2002.

[HHNS02] A. Hagerer, H. Hungar, O. Niese, and B. Steffen. Model generation by moderated regular extrapolation. In R.-D. Kutsche and H. Weber, editors, Proc. FASE02, volume 2306 of LNCS, pages 80-95. Springer Verlag, 2002. 
$\left[\mathrm{HLN}^{+}\right.$90] D. Harel, H. Lachover, A. Naamad, A. Pnueli, M. Politi, R. Sherman, A. Shtull-Trauring, and M.B. Trakhtenbrot. STATEMATE: A working environment for the development of complex reactive systems. IEEE Trans. on Software Engineering, 16(4):403-414, April 1990.

[HMP94] T.A. Henzinger, Z. Manna, and A. Pnueli. Temporal proof methodologies for timed transition systems. Information and Computation, 112:173-337, 1994.

[HNS03] Hardi Hungar, Oliver Niese, and Bernhard Steffen. Domain-specific optimization in automata learning. In Proc. CAV'03, 2003.

[Hol00] G.J. Holzmann. Logic verification of ANSI-C code with SPIN. In K. Havelund, J. Penix, and W. Visser, editors, SPIN Model Checking and Software Verification: Proc. $7^{\text {th }}$ Int. SPIN Workshop, volume 1885 of LNCS, pages 131-147, Stanford, CA, 2000. Springer Verlag.

[HRS98] T.A. Henzinger, J.-F. Raskin, and P.-Y. Schobbens. The regular real-time languages. In K.G. Larsen, S. Skuym, and G. Winskel, editors, ICALP98, volume 1443 of $L N C S$, pages 580-591. Springer Verlag, 1998.

[KV94] M.J. Kearns and U.V. Vazirani. An Introduction to Computational Learning Theory. MIT Press, 1994.

[LMS04] François Laroussinie, Nicolas Markey, and Ph. Schnoebelen. Model checking timed automata with one or two clocks. In Proceedings of 15th International Conference on Concurrency Theory, pages 387401, 2004.

[MP04] O. Maler and A. Pnueli. On recognizable timed languages. In Proc. FOSSACS04, LNCS. Springer-Verlag, 2004.

[Od195] A. M. Odlyzko. Asymptotic enumeration methods. In Handbook of Combinatorics, volume 2, pages 1063-1229. North-Holland, Amsterdam, 1995.

[RS93] R.L. Rivest and R.E. Schapire. Inference of finite automata using homing sequences. Information and Computation, 103:299-347, 1993.

[SEG00] M. Schmitt, M. Ebner, and J. Grabowski. Test generation with autolink and testcomposer. In Proc. 2nd Workshop of the SDL Forum Society on SDL and MSC - SAM'2000, June 2000.

[SV96] Jan Springintveld and Frits Vaandrager. Minimizable timed automata. In B. Jonsson and J. Parrow, editors, Proc. FTRTFT'96, volume 1135 of LNCS, pages 130-147. Springer Verlag, 1996. 
[SVD01] Jan Springintveld, Frits W. Vaandrager, and Pedro R. D'Argenio. Testing timed automata. Theor. Comput. Sci., 254(1-2):225-257, 2001.

[TB73] B.A. Trakhtenbrot and J.M. Barzdin. Finite automata: behaviour and synthesis. North-Holland, 1973.

[Vas73] M. P. Vasilevskii. Failure diagnosis of automata. Cybernetic, 9(4):653-665, 1973.

[VdWW06] Sicco E. Verwer, Mathijs M. de Weerdt, and Cees Witteveen. Identifying an automaton model for timed data. In Proceedings of the Annual Machine Learning Conference of Belgium and the Netherlands (Benelearn), pages 57-64. Benelearn, 2006.

[Wil94] T. Wilke. Specifying timed state sequences in powerful decidable logics and timed automata. In H Langmaack, W. P. de Roever, and J. Vytopil, editors, Proc. FTRTFT'94, volume 863 of LNCS, pages 694-715. Springer Verlag, 1994. 